\title{
Quantum computing with antiferromagnetic spin clusters
}

\author{
Florian Meier ${ }^{1}$, Jeremy Levy ${ }^{2,3}$, and Daniel Loss ${ }^{1,3}$ \\ ${ }^{1}$ Department of Physics and Astronomy, University of Basel, Klingelbergstrasse 82, 4056 Basel, Switzerland \\ ${ }^{2}$ Department of Physics and Astronomy, University of Pittsburgh, Pittsburgh, Pennsylvania 15260 \\ ${ }^{3}$ Center for Oxide-Semiconductor Materials for Quantum Computation, Pittsburgh, Pennsylvania 15260
}

(Dated: June 19, 2018)

\begin{abstract}
We show that a wide range of spin clusters with antiferromagnetic intracluster exchange interaction allows one to define a qubit. For these spin cluster qubits, initialization, quantum gate operation, and readout are possible using the same techniques as for single spins. Quantum gate operation for the spin cluster qubit does not require control over the intracluster exchange interaction. Electric and magnetic fields necessary to effect quantum gates need only be controlled on the length scale of the spin cluster rather than the scale for a single spin. Here, we calculate the energy gap separating the logical qubit states from the next excited state and the matrix elements which determine quantum gate operation times. We discuss spin cluster qubits formed by one- and twodimensional arrays of $s=1 / 2$ spins as well as clusters formed by spins $s>1 / 2$. We illustrate the advantages of spin cluster qubits for various suggested implementations of spin qubits and analyze the scaling of decoherence time with spin cluster size.
\end{abstract}

\section{INTRODUCTION}

During the past years, the discovery of several powerful quantum algorithms 1 has triggered substantial research efforts aiming at the implementation of a quantum computer in a physical system. The main difficulty is that qubits must be prepared, manipulated, and read out with high fidelity while decoherence is required to remain small ${ }^{2}$ Solid-state implementations of qubits exploit the versatility of nanoscale fabrication, but suffer from decoherence times which are usually shorter than in many quantum optics proposals ${ }^{3}$ Electron 4.5 .6 and nuclear ${ }^{7.8}$ spins have been identified as promising candidates for qubits in a solid state system. The main advantage of electron or nuclear spins is that they are natural two state systems and that decoherence times for the spin degree of freedom ${ }^{9,10}$ are usually larger than for charge degrees of freedom.

Here we show that a wide variety of spin clusters are promising candidate systems for qubits. Qubits formed by several spins have so far mainly been discussed in the context of exchange-only quantum computing, $11,12,13$ coherence-preserving qubits 14 , and quantum computing schemes in which the requirements on the control of exchange interactions between spins are relaxed ${ }^{15}$ However, all these schemes require control at the single-spin level, either with local magnetic fields 15 or exchange interactions 11.14 For the spin clusters considered here, control for both magnetic fields and exchange interactions is required only on the length scale of the spin cluster diameter. As we have shown in Ref. 16, spin chains formed by an odd number of antiferromagnetically coupled spins $s=1 / 2$ allow one to define a logical qubit. The logical state of the qubit is encoded in the collective state of the spin cluster. Here we detail that this construction remains valid for a wide range of spin clusters, independent of the details of intracluster exchange interaction and spin placement. Initialization and readout of the spin cluster is achieved with the methods developed for single spins ${ }^{4.5}$ The main advantage of spin clusters is that the requirements on spatial control can be traded for gate operation times. The scaling of the decoherence rate with the size of the spin cluster depends on the microscopic decoherence mechanism. While the decoherence rate induced by fluctuating local magnetic fields increases with cluster size, we show that magnetic dipolar interactions for the spin cluster qubit are smaller than for single spins. The optimum size of the spin cluster qubit is determined by a trade-off between the increase in gate operation times and the decoherence rate effected by local fluctuating magnetic fields, the decrease in magnetic dipolar interaction energy, and the relaxed conditions on local control.

Any quantum computation can be decomposed into a sequence of one- and two-qubit quantum gates ${ }^{17}$ For a single-spin qubit, the $\hat{s}_{z}$ eigenstates $|\uparrow\rangle$ and $|\downarrow\rangle$ are identified as logical basis states $|0\rangle$ and $|1\rangle$, respectively ${ }^{4.5}$ The phase shift gate can then be realized by a magnetic field $B_{z}(t)$ and the one-qubit rotation gate $U_{\text {rot }}$ by a transverse field $B_{x}(t)$ which rotates $|\uparrow\rangle$ into $|\downarrow\rangle$ and vice versa. More generally, the equations

$$
\left\langle 0\left|\hat{H}^{\prime}\right| 0\right\rangle=\left\langle 1\left|\hat{H}^{\prime}\right| 1\right\rangle \text { and }\left\langle 1\left|\hat{H}^{\prime}\right| 0\right\rangle \neq 0
$$

constitute a sufficient condition that a Hamiltonian $\hat{H}^{\prime}$ induces the unitary time evolution required for $U_{\text {rot }}$. For single spins, $\hat{H}^{\prime}=g \mu_{B} B_{x}(t) \hat{s}_{x}$ fulfills Eq. (1). Similarly, an exchange interaction $\hat{H}_{*}=J_{*} \hat{\mathbf{s}}_{1} \cdot \hat{\mathbf{s}}_{2}$ generates the unitary time evolution required for the square-root of SWAP gate ${ }^{4}$ because, in the two-qubit product basis,

$$
\left\langle 10\left|\hat{H}_{*}\right| 01\right\rangle \neq 0 \text {. }
$$

In contrast to a single spin $s=1 / 2$, clusters formed by $n_{c}$ coupled spins are not intrinsically two-state systems. In order to prove that a logical qubit can be defined in terms of the energy eigenstates of a spin cluster we will (a) identify spin clusters with a ground state doublet $\{|0\rangle,|1\rangle\}$ separated from the next excited state by an energy gap 
$\Delta$; (b) identify Hamiltonians $\hat{H}^{\prime}$ and $\hat{H}_{*}$ which satisfy Eqs. (11) and (2) and, hence, allow one to generate a universal set of quantum gates; and (c) quantify leakage and decoherence for the spin cluster qubit. In particular, the evaluation of the matrix elements in Eqs. (11) and (2) and the quantification of excitation out of the computational basis (leakage) requires a detailed characterization of the states $\{|0\rangle,|1\rangle\}$ which is, in general, nontrivial.

This paper is organized as follows. In Sec. II we discuss the computational basis states for spin- $1 / 2$ chains. For this simple geometry, it is possible to derive analytical expressions for the matrix elements in Eqs. (11) and (2) for various anisotropies and spatially varying intracluster exchange interaction. Section III discusses the insensitivity of spin cluster qubits to the details of interactions within the cluster, such as the relative placement of spins and the exchange strengths. In Sec.IV spins with spin quantum numbers larger than $1 / 2$ are discussed. In Sec. V we draw our conclusions.

\section{SPIN CHAINS}

For simplicity, we first consider a spin cluster qubit formed by a spin chain,

$$
\hat{H}=\sum_{i=1}^{n_{c}-1} f_{j}\left[J_{\perp}\left(\hat{s}_{j, x} \hat{s}_{j+1, x}+\hat{s}_{j, y} \hat{s}_{j+1, y}\right)+J_{z} \hat{s}_{j, z} \hat{s}_{j+1, z}\right]
$$

where $n_{c}$ is odd and $J_{\perp}, J_{z}>0$. The real numbers $f_{j}>0$ account for a spatial variation of the exchange interaction, and $J_{\perp} f_{j}\left(J_{z} f_{j}\right)$ denotes the transverse (longitudinal) exchange interaction between sites $j$ and $j+1$.

\section{A. Isotropic spin chains}

For electron spins in quantum dots, the nearest neighbor exchange is usually of the Heisenberg form, $J=$ $J_{\perp}=J_{z}$. We first consider $f_{j} \equiv 1$,

$$
\hat{H}=J \sum_{j=1}^{n_{c}-1} \hat{\mathbf{s}}_{j} \cdot \hat{\mathbf{s}}_{j+1}
$$

with $J>0$. Note that this is an open spin chain; a closed spin chain would have a fourfold degenerate ground state multiplet for odd $n_{c}$ that would make it unsuitable for representing a single qubit. Because the intracluster exchange interaction $J$ is time independent and no external control is required, $J$ can be adjusted already during sample growth.

Spin chains have been studied in great detail during the past decades $\frac{18.19 .20}{10}$ The theoretical description of the antiferromagnetic spin chain Eq. (44) is particularly challenging because the classical Néel ordered state is not an energy eigenstate and quantum fluctuations are

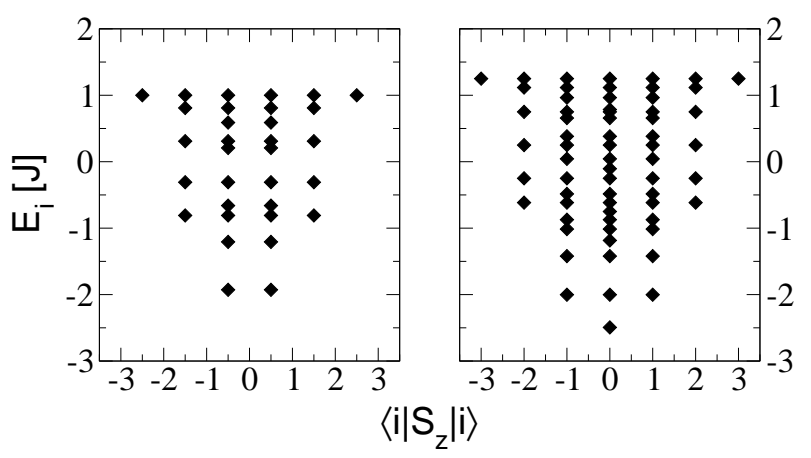

FIG. 1: Energy spectrum of an isotropic spin chain with $n_{c}=$ 5 (left panel) and $n_{c}=6$ (right panel). Energy eigenstates are sorted according to their quantum numbers of $\hat{S}_{z}$ and their eigenenergies.

pronounced. We define the operator of total spin,

$$
\hat{S}_{\alpha}=\sum_{j=1}^{n_{c}} \hat{s}_{j, \alpha}
$$

for $\alpha=x, y, z$. Energy eigenstates can be labeled according to their quantum numbers of total spin $\hat{\mathbf{S}}$ and the $z$-component of total spin, $\hat{S}_{z}$, because

$$
\left[\hat{H}, \hat{\mathbf{S}}^{2}\right]=\left[\hat{H}, \hat{S}_{z}\right]=0 .
$$

Due to the antiferromagnetic exchange coupling, states in which the total spin of the chain is minimized are energetically most favorable ${ }^{21}$ For even $n_{c}$, the minimum possible spin is $S=0$, and the system has a nondegenerate ground state. In contrast, for odd $n_{c}$, there is a ground state doublet (Fig. 11) ${ }^{21}$ This parity effect is well known for thermodynamic quantities ${ }^{22}$ The energy gap $\Delta$ separating the ground state doublet from the next excited state,

$$
\Delta \simeq \frac{J \pi}{2} k_{\min } \sim \frac{J \pi^{2}}{2 n_{c}}
$$

can be estimated from the lower bound of the des Cloiseaux-Pearson spectrum and the minimum wave vector $k_{\min }=\pi / n_{c}$ (Ref. 23). Henceforth, we will restrict our attention to spin chains with odd $n_{c}$.

The requirements on a candidate system for qubits include initialization of the quantum computer, a universal set of quantum gates, decoherence times long compared to gate operation times, and readout of the qubit $\stackrel{2}{2}^{2}$

\section{Definition of the spin cluster qubit}

For the chain with an odd number of sites [Fig. 2(a)], we define the spin cluster qubit in terms of the $S=1 / 2$ ground state doublet by

$$
\begin{aligned}
\hat{S}_{z}|0\rangle & =\frac{1}{2}|0\rangle, \\
\hat{S}_{z}|1\rangle & =-\frac{1}{2}|1\rangle .
\end{aligned}
$$




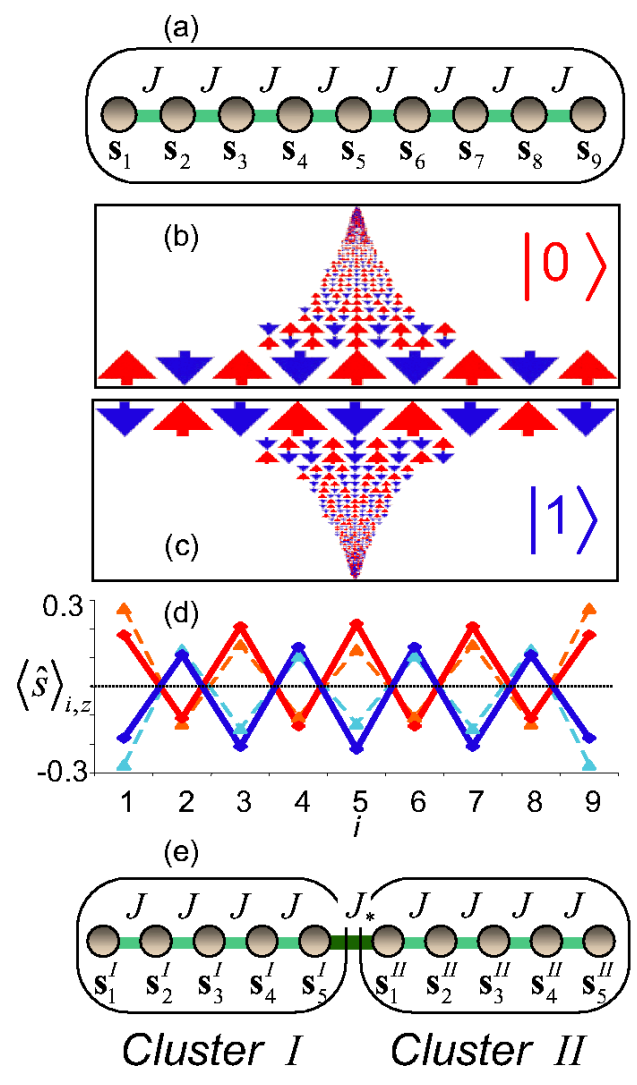

FIG. 2: Spin cluster qubit. (a) The energy eigenstates of an antiferromagnetic spin chain with an odd number of sites define the spin cluster qubit. (b) Wave function of $|0\rangle$ in the single-spin product basis for $n_{c}=9$. The size of each configuration is proportional to the probability of finding the corresponding product state in $|0\rangle$. (c) Similar to (b) but for state $|1\rangle$. (d) Spin density $\left\langle\psi\left|\hat{s}_{i, z}\right| \psi\right\rangle$ for $|\psi\rangle=|0\rangle$ and $|\psi\rangle=|1\rangle$ (solid lines) for constant intrachain exchange coupling. Dashed lines indicate the corresponding data for spatially varying intrachain exchange with $f_{j}=\sin \left(j \pi / n_{c}\right)$. (e) Spin cluster qubits are coupled by a switchable interchain exchange coupling $J_{*}(t)$.

The states $\{|0\rangle,|1\rangle\}$ do not in general have a simple representation in the single-spin product basis, but rather are complicated superpositions of $n_{c} ! /\left[\left(n_{c}-1\right) / 2\right] !\left[\left(n_{c}+1\right) / 2\right]$ ! states [Figs. [2 b) and (c)] as evidenced by the local magnetization density [Fig. 2(d)]. The largest amplitude in this superposition corresponds to the Néel ordered states $|\uparrow\rangle_{1}|\downarrow\rangle_{2} \ldots|\uparrow\rangle_{n_{c}}(|0\rangle)$ and $|\downarrow\rangle_{1}|\uparrow\rangle_{2} \ldots|\downarrow\rangle_{n_{c}}(|1\rangle)$, respectively. For $n_{c}=9$, the Néel configuration has only a $20 \%$ probability; the remaining $80 \%$ represent quantum fluctuations [see Figs. 2(b) and (c)].

In spite of their complicated representation in the single-spin product basis, $|0\rangle$ and $|1\rangle$ are in many respects very similar to the states $|\uparrow\rangle$ and $|\downarrow\rangle$ of a single spin and, hence, define the computational basis for universal quantum computing with spin cluster qubits. The reason for this is that $\{|0\rangle,|1\rangle\}$ belong to one $S=1 / 2$ doublet such that

$$
\hat{S}^{-}|0\rangle=|1\rangle, \quad \hat{S}^{+}|1\rangle=|0\rangle,
$$

where $\hat{S}^{ \pm}=\hat{S}_{x} \pm i \hat{S}_{y}$ are the spin ladder operators of the spin chain.

\section{Initialization}

Initialization of the spin cluster qubit can be achieved by cooling in a magnetic field with $g \mu_{B} B_{z} \ll \Delta$ to temperatures $T \ll g \mu_{B} B_{z} / k_{B}$. The spin cluster will relax to $|0\rangle$ within the spin relaxation time. Because thermalization is typically slow, the preparation of a given initial state could be facilitated by the measurement of the spins within the clusters, possibly followed by local operations. In this way, a state which is energetically close to the ground state could be prepared.

\section{Quantum Gate operation}

The one-qubit phase shift gate $U_{\phi}$, one-qubit rotation gate $U_{\text {rot }}$, and CNOT gate $U_{\mathrm{CNOT}}$ constitute a universal set of quantum gates. According to Eq. (9), a magnetic field that is constant over the spin cluster qubit acts on the states of the spin cluster qubit in the same way as on a single-spin qubit 16 Constant magnetic fields $B_{z}\left(B_{x}\right)$ effect the one-qubit phase shift (one-qubit rotation) quantum gate without leakage. Because $|0\rangle$ and $|1\rangle$ are degenerate and separated from the next excited state by $\Delta$, one-qubit quantum gates can be realized with high fidelity also by any spatially varying magnetic field for which $\left|\left\langle 1\left|\sum_{j=1}^{n_{c}} g_{j} \mu_{B} B_{j, x} \hat{s}_{j, x}\right| 0\right\rangle\right| \neq 0\left(U_{\text {rot }}\right)$ and $\left\langle 0\left|\sum_{j=1}^{n_{c}} g_{j} \mu_{B} B_{j, z} \hat{s}_{j, z}\right| 0\right\rangle \neq 0\left(U_{\phi}\right)$, respectively. Here, $g_{j}$ is the electron spin $g$ factor at site $j$. Such spatially varying fields can potentially cause leakage. However, if $\left|g_{j} \mu_{B} B_{j}\right| / \Delta \ll 1$ and if all $B_{j}$ are switched on and off adiabatically, i.e., on time scales long compared to $h / \Delta$, the quantum gate fidelity is close to $100 \%$ as we will discuss in detail in Sec. IIC

For the CNOT gate, one requires an exchange interaction $\hat{H}_{*}$ between one or several spins of neighboring spin cluster qubits I and II which can be switched on and off, e.g., by electrical gates $\underline{\underline{4}}$ The simplest case of an exchange interaction between the outermost spins of neighboring clusters [Fig. [2(e)], $\hat{H}_{*}=J_{*}(t) \hat{\mathbf{s}}_{n_{c}}^{\mathrm{I}} \cdot \hat{\mathbf{s}}_{1}^{\mathrm{II}}$ translates into an isotropic exchange interaction also for the two-qubit product basis. This remains true for any $\hat{H}_{*}$ of the form

$$
\hat{H}_{*}=J_{*}(t) \hat{\mathbf{s}}_{n_{c}}^{\mathrm{I}} \cdot \hat{\mathbf{s}}_{1}^{\mathrm{II}}+J_{*}(t) \sum_{j=1}^{n_{c}-1}\left(v_{j}^{\mathrm{I}} \hat{\mathbf{s}}_{j}^{\mathrm{I}} \cdot \hat{\mathbf{s}}_{j+1}^{\mathrm{I}}+v_{j}^{\mathrm{II}} \hat{\mathbf{s}}_{j}^{\mathrm{II}} \cdot \hat{\mathbf{s}}_{j+1}^{\mathrm{II}}\right) .
$$

Here, the factors $v_{j}$ allow for a spatial variation of the intracluster exchange coupling constants during gate operation, where $\left|v_{j}\right|<1$ and $\left|J_{*}\right| \ll J$ is the limit relevant 
for experiments. $\hat{H}_{*}$ will in general not only couple states within the two-qubit product basis $\{|00\rangle,|01\rangle,|10\rangle,|11\rangle\}$, but will also lead to leakage. As long as $J_{*}(t)$ changes adiabatically and $\left|J_{*}(t)\right| \ll \Delta$ for all times, leakage remains small. It should be noted that this adiabaticity condition also holds for single electrons in quantum dots where, however, the energy gap $\Delta$ is usually larger than for the spin cluster qubit. The action of $\hat{H}_{*}$ can then be described by an effective Hamiltonian in the two-qubit product basis,

$$
\begin{gathered}
\hat{H}_{*}=J_{* z}(t) \hat{S}_{z}^{\mathrm{I}} \hat{S}_{z}^{\mathrm{II}}+\frac{J_{* \perp}(t)}{2}\left(\hat{S}^{\mathrm{I}+} \hat{S}^{\mathrm{II}-}+\hat{S}^{\mathrm{I}-} \hat{S}^{\mathrm{II}+}\right) \\
+J_{o}(t) \mathbf{1}
\end{gathered}
$$

where the roman numbers label the spin clusters, and

$$
\begin{aligned}
J_{* z}(t) & =\left.\left.4 J_{*}(t)\right|_{\mathrm{I}}\left\langle 0\left|\hat{s}_{n_{c}, z}^{\mathrm{I}}\right| 0\right\rangle_{\mathrm{I}}\right|_{\mathrm{II}}\left\langle 0\left|\hat{s}_{1, z}^{\mathrm{II}}\right| 0\right\rangle_{\mathrm{II}} \mid, \\
J_{* \perp}(t)= & \left.\left.4 J_{*}(t)\right|_{\mathrm{I}}\left\langle 1\left|\hat{s}_{n_{c}, x}^{\mathrm{I}}\right| 0\right\rangle_{\mathrm{I}}\right|_{\mathrm{II}}\left\langle 1\left|\hat{s}_{1, x}^{\mathrm{II}}\right| 0\right\rangle_{\mathrm{II}} \mid, \\
J_{O}(t)= & J_{*}(t)\left[{ }_{\mathrm{I}}\left\langle 0\left|\sum_{j=1}^{n_{c}-1} v_{j}^{\mathrm{I}} \hat{\mathbf{s}}_{j}^{\mathrm{I}} \cdot \hat{\mathbf{s}}_{j+1}^{\mathrm{I}}\right| 0\right\rangle_{\mathrm{I}}\right. \\
& \left.+{ }_{\mathrm{II}}\left\langle 0\left|\sum_{j=1}^{n_{c}-1} v_{j}^{\mathrm{II}} \hat{\mathbf{s}}_{j}^{\mathrm{II}} \cdot \hat{\mathbf{s}}_{j+1}^{\mathrm{II}}\right| 0\right\rangle_{\mathrm{II}}\right] .
\end{aligned}
$$

For the derivation of Eq. (11), see Appendix A

Because, for the isotropic chain,

$$
{ }_{\mathrm{I}}\left\langle 0\left|\hat{s}_{n_{c}, z}^{\mathrm{I}}\right| 0\right\rangle_{\mathrm{I}}={ }_{\mathrm{I}}\left\langle 1\left|\hat{s}_{n_{c}, x}^{\mathrm{I}}\right| 0\right\rangle_{\mathrm{I}} \mid,
$$

the coupling $\hat{H}_{*}$ is isotropic also in the two-qubit product basis and acts on the states $|0\rangle$ and $|1\rangle$ of neighboring spin chains in the same way as an isotropic exchange interaction between two single spins.

An explicit switching sequence for the CNOT gate based on single-qubit rotations and the unitary time evolution governed by the exchange interaction in Eq. (11) is 16

$$
\begin{aligned}
& U_{\mathrm{CNOT}} \sim e^{-i \pi S_{y}^{\mathrm{II}} / 2} e^{i 2 \pi \mathbf{n}_{1} \cdot \mathbf{S}^{\mathrm{I}} / 3} e^{i 2 \pi \mathbf{n}_{2} \cdot \mathbf{S}^{\mathrm{II}} / 3} U_{*}(\pi / 2) \\
& \quad \times e^{i \pi S_{y}^{\mathrm{I}}} U_{*}(\pi / 2) e^{-i \pi S_{x}^{\mathrm{I}} / 2} e^{-i \pi S_{x}^{\mathrm{II}} / 2} e^{i \pi S_{y}^{\mathrm{II}} / 2}
\end{aligned}
$$

for the general case where $J_{* z} \neq J_{* \perp}$. Here, $\mathbf{n}_{1}=$ $(1,-1,1) / \sqrt{3}$ and $\mathbf{n}_{2}=(1,1,-1) / \sqrt{3}$, and we have defined the unitary time evolution operator $U_{*}(\pi / 2)=$ $\hat{\mathrm{T}}_{t} \exp \left(-i \int d t \hat{H}_{*} / \hbar\right)$, with $-\int d t J_{* \perp}(t) / \hbar=\pi / 2$.

The gate operation time for $U_{*}(\pi / 2)$ is limited from below by $h /\left.\left.16 J_{*}\right|_{\mathrm{I}}\left\langle 1\left|\hat{s}_{n_{c}, x}^{\mathrm{I}}\right| 0\right\rangle_{\mathrm{I}}\right|_{\mathrm{II}}\left\langle 1\left|\hat{s}_{1, x}^{\mathrm{II}}\right| 0\right\rangle_{\mathrm{II}} \mid$, where $J_{*}$ is the maximum value of the exchange coupling. Matrix elements such as $\left.\right|_{\mathrm{I}}\left\langle 1\left|\hat{s}_{n_{c}, x}^{\mathrm{I}}\right| 0\right\rangle_{\mathrm{I}} \mid$ decrease with increasing $n_{c}$, which leads to an increase in gate operation time. For realistic parameters and small $n_{c}$ (see Sec. IIF below), $J_{*}$ is limited by experimental constraints rather than the condition $J_{*} \ll \Delta$. Then, the increase in gate operation time compared to single-spin qubits is $1 /\left(\left.\left.4\right|_{\mathrm{I}}\left\langle 1\left|\hat{s}_{n_{c}, x}^{\mathrm{I}}\right| 0\right\rangle_{\mathrm{I}}\right|_{\mathrm{II}}\left\langle 1\left|\hat{s}_{1, x}^{\mathrm{II}}\right| 0\right\rangle_{\mathrm{II}} \mid\right)$ and depends only on the matrix elements of the spin operators. Similarly, for a given magnetic field, the increase of the time required for a one-qubit rotation depends only on the matrix elements $\left|\left\langle 1\left|\hat{s}_{j, x}\right| 0\right\rangle\right|$.

\section{Decoherence}

For spin clusters, decoherence usually is faster than for single spins. The scaling of the decoherence time $\tau_{\phi}$ with system size depends on the microscopic decoherence mechanism. For electron spins in quantum dots, fluctuating fields and nuclear spins have been identified as dominant sources $4,5,24,25,26,27,28$ In the following, we discuss two scenarios for decoherence in which (i) a fluctuating field couples to the total magnetic moment of the spin cluster qubit, and (ii) independent fluctuating fields act on each spin of the spin cluster qubit individually.

In order to obtain analytical estimates for the scaling of $\tau_{\phi}$ with $n_{c}$, we restrict our analysis to a phenomenological model in which decoherence is effected by a fluctuating classical field $\hbar b(t)$. In case (i),

$$
\hat{H}^{B}=\hbar b(t) \hat{S}_{z}
$$

The decoherence rate is obtained from 29

$$
\begin{aligned}
\frac{1}{\tau_{\phi}}= & \pi \sum_{|k\rangle \neq|0\rangle}\left|\left\langle k\left|\hat{S}_{z}\right| 0\right\rangle\right|^{2} C\left(E_{k}-E_{0}\right) \\
& +\pi \sum_{|k\rangle \neq|1\rangle}\left|\left\langle k\left|\hat{S}_{z}\right| 1\right\rangle\right|^{2} C\left(E_{k}-E_{1}\right) \\
& +\pi\left(\left\langle 0\left|\hat{S}_{z}\right| 0\right\rangle-\left\langle 1\left|\hat{S}_{z}\right| 1\right\rangle\right)^{2} C(0),
\end{aligned}
$$

where

$$
C(E)=\frac{1}{2 \pi} \int_{-\infty}^{\infty} d t e^{i E t / \hbar}\langle b(t) b(0)\rangle
$$

is the spectral density of the random field in Eq. (15). Because of Eq. (8), we find that the decoherence rate $1 / \tau_{\phi}=\pi C(0)$ is independent of $n_{c}$. This result, which is in stark contrast to the standard result that the decoherence rate increases with system size, can be traced back to the fact that in Eq. (15) only the total magnetic moment couples to the fluctuating field and the magnetic moment $\pm g \mu_{B} / 2$ of the spin cluster qubit is identical to the one of a single spin. Similarly, for a nondiagonal coupling $\hat{H}^{B}=\hbar b(t) \hat{S}_{x}$, from Eq. (16) we find $1 / \tau_{\phi}=\pi C(0) / 2$.

Decoherence due to the coupling to nuclear spins is a complicated theoretical problem in its own right $24,25,26,27,28$ In order to obtain a heuristic estimate for the scaling of the decoherence time with $n_{c}$ for this decoherence scenario, we consider fluctuating classical fields which act independently on each site of the spin cluster,

$$
\hat{H}^{B}=\sum_{j=1}^{n_{c}} \hbar b_{j}(t) \hat{s}_{j, z},
$$


where $\left\langle b_{i}(t) b_{j}(0)\right\rangle \propto \delta_{i j}$. For Gaussian white noise with

$$
\left\langle b_{i}(t) b_{j}(0)\right\rangle=2 \pi \gamma^{B} \delta_{i j} \delta(t),
$$

$1 / \tau_{\phi} \simeq n_{c} \pi \gamma^{B}$ scales linearly with $n_{c}$, i.e., if fluctuating fields act independently on the individual spins of the cluster, the decoherence rate increases with $n_{c}$. This result for the scaling of $1 / \tau_{\phi}$ with system size agrees with the standard result for $n_{c}$ qubits which are subject to independently fluctuating fields ${ }^{30}$ Note that an increase in the decoherence rate by a factor $n_{c}$ implies that the probability for the state of the spin cluster qubit to remain unaffected by the fluctuating fields for a certain time $t$ decreases exponentially with $n_{c}$ (Ref. 30).

Magnetic dipolar interactions are another source of decoherence. Consider two single-spin qubits $\hat{\mathbf{s}}_{1}$ and $\hat{\mathbf{s}}_{2}$ with coordinate vectors $\mathbf{r}_{1}$ and $\mathbf{r}_{2}$, respectively, that are coupled by the magnetic dipolar interaction,

$$
\hat{H}_{\text {dip }}=\frac{\mu_{0}\left(g \mu_{B}\right)^{2}}{4 \pi} \frac{\hat{\mathbf{s}}_{1} \cdot \hat{\mathbf{s}}_{2}-3\left(\hat{\mathbf{s}}_{1} \cdot \mathbf{e}_{r}\right)\left(\hat{\mathbf{s}}_{2} \cdot \mathbf{e}_{r}\right)}{d^{3}},
$$

where $\mathbf{r}=\mathbf{r}_{1}-\mathbf{r}_{2}, d=|\mathbf{r}|$ is the distance between the single-spin qubits, and $\mathbf{e}_{r}=\mathbf{r} /|\mathbf{r}|$. In first order perturbation theory, the dipolar interaction leads to an energy shift $\pm E_{\text {dip }}$ for the product states with parallel and antiparallel spin configurations, respectively. For lateral quantum dots with spin quantization axis perpendicular to $\mathbf{e}_{r}$,

$$
\begin{aligned}
E_{\text {dip }} & =\left\langle\uparrow, \uparrow\left|\hat{H}_{\text {dip }}\right| \uparrow, \uparrow\right\rangle \\
& =\frac{\mu_{0}\left(g \mu_{B}\right)^{2}}{4 \pi} \frac{\left\langle\uparrow, \uparrow\left|\hat{\mathbf{s}}_{1} \cdot \hat{\mathbf{s}}_{2}\right| \uparrow, \uparrow\right\rangle}{d^{3}}=\frac{\mu_{0}\left(g \mu_{B}\right)^{2}}{16 \pi d^{3}}(21)
\end{aligned}
$$

because the expectation value of $\left(\hat{\mathbf{s}}_{1} \cdot \mathbf{e}_{r}\right)\left(\hat{\mathbf{s}}_{2} \cdot \mathbf{e}_{r}\right)$ vanishes identically. Similarly, we find

$$
\begin{aligned}
& \left\langle\downarrow, \downarrow\left|\hat{H}_{\text {dip }}\right| \downarrow, \downarrow\right\rangle=+E_{\text {dip }}, \\
& \left\langle\downarrow, \uparrow\left|\hat{H}_{\text {dip }}\right| \downarrow, \uparrow\right\rangle=-E_{\text {dip }}, \\
& \left\langle\uparrow, \downarrow\left|\hat{H}_{\text {dip }}\right| \uparrow, \downarrow\right\rangle=-E_{\text {dip }} .
\end{aligned}
$$

The deterministic phase shift due to the different dipolar energies for parallel and antiparallel spin configurations could be accounted for at the end of a quantum algorithm if no other error sources were present. However, it leads to correlated errors when spins are coupled to an environment that induces, e.g., spin flip errors. While $E_{\text {dip }}$ is too small to induce errors of order $10^{-4}$ for electron spins in quantum dots, dipolar interactions are large for, e.g., $\mathrm{P}$ dopants in a Si matrix (see Sec. IIF). We show next that dipolar interactions for spin cluster qubits are smaller than for single-spin qubits. For definiteness, we consider two clusters with spins at sites $\mathbf{r}_{j}^{\mathrm{I}}=j d \mathbf{e}_{y}$ and $\mathbf{r}_{j}^{\mathrm{II}}=\left(j+n_{c}\right) d \mathbf{e}_{y}$ for clusters I and II, respectively, with $j=1,2, \ldots, n_{c}$ [Fig. [2(c)]. The intracluster dipolar interactions lead to an unimportant energy shift that is identical for both $|0\rangle$ and $|1\rangle$,

$$
\sum_{i>j} \frac{\mu_{0}\left(g \mu_{B}\right)^{2}}{4 \pi d^{3}}\left\langle 0\left|\frac{\hat{\mathbf{s}}_{i} \cdot \hat{\mathbf{s}}_{j}-3 \hat{s}_{i, y} \hat{s}_{j, y}}{(i-j)^{3}}\right| 0\right\rangle
$$

$$
=\sum_{i>j} \frac{\mu_{0}\left(g \mu_{B}\right)^{2}}{4 \pi d^{3}}\left\langle 1\left|\frac{\hat{\mathbf{s}}_{i} \cdot \hat{\mathbf{s}}_{j}-3 \hat{s}_{i, y} \hat{s}_{j, y}}{(i-j)^{3}}\right| 1\right\rangle
$$

because of symmetry. The interqubit dipolar interaction,

$$
\hat{H}_{\mathrm{dip}}^{\mathrm{sc}}=\frac{\mu_{0}\left(g \mu_{B}\right)^{2}}{4 \pi d^{3}} \sum_{i, j} \frac{\hat{\mathbf{s}}_{i}^{\mathrm{I}} \cdot \hat{\mathbf{s}}_{j}^{\mathrm{II}}-3 \hat{s}_{i, y}^{\mathrm{I}} \hat{s}_{j, y}^{\mathrm{II}}}{\left(i-j+n_{c}\right)^{3}},
$$

gives rise to an energy shift of the states $|00\rangle$ and $|11\rangle$ relative to $|01\rangle$ and $|10\rangle$, where $|0\rangle$ and $|1\rangle$ denote the logical basis of the spin cluster qubit,

$$
\begin{aligned}
& \left\langle 00\left|\hat{H}_{\mathrm{dip}}^{\mathrm{sc}}\right| 00\right\rangle=\left\langle 11\left|\hat{H}_{\mathrm{dip}}^{\mathrm{sc}}\right| 11\right\rangle=E_{\mathrm{dip}}^{\mathrm{sc}}, \\
& \left\langle 01\left|\hat{H}_{\mathrm{dip}}^{\mathrm{sc}}\right| 01\right\rangle=\left\langle 10\left|\hat{H}_{\mathrm{dip}}^{\mathrm{sc}}\right| 10\right\rangle=-E_{\mathrm{dip}}^{\mathrm{sc}},
\end{aligned}
$$

similarly to single-spin qubits. Evaluating the matrix elements numerically, we find that the characteristic dipolar energy $E_{\text {dip }}^{\mathrm{sc}}$ decreases with increasing $n_{c}$ We find $E_{\text {dip }}^{\text {sc }} / E_{\text {dip }}=0.42,0.25,0.16$, and 0.12 for $n_{c}=3,5,7$, and 9 , respectively, where $E_{\text {dip }}$ is the dipolar energy for single-spin qubits. This shows that decoherence effected by magnetic dipolar interactions is indeed smaller for spin cluster qubits than for single spins.

\section{Readout}

Readout of the spin cluster qubit is achieved by measuring the individual spins within the cluster $\left(\hat{s}_{i, z}\right)$ or the state of the total spin of the cluster $\left(\hat{S}_{z}\right)$. The measurement of individual spins still is a considerable experimental challenge. However, as has been shown theoretically $\stackrel{4.31}{.}$ measurement of single spins is feasible via charge degrees of freedom. More specifically, the state of a single spin on a quantum dot can be detected by a current flowing between spin polarized leads that are tunnel coupled to the quantum dot 31

If an experimental technique is established that allows one to measure the state of a single spin, it will also be possible to measure the state of a spin cluster qubit by measurement of all spins of the cluster. Because $\left\langle 0\left|\sum_{i=1}^{n_{c}} \hat{s}_{i, z}\right| 0\right\rangle=1 / 2$ and $\left\langle 1\left|\sum_{i=1}^{n_{c}} \hat{s}_{i, z}\right| 1\right\rangle=-1 / 2$, this will allow one to unambiguously determine the state of the cluster qubit. However, the state of the cluster determines the local spin density at each site [Fig. 2(d)], and a probabilistic readout is possible also by measurement of single spins only. For example, for $n_{c}=9$, if the measurement of the central spin of the chain yields $+1 / 2$, the spin cluster qubit has been in state $|0\rangle$ with a probability of $70 \%$. A selective readout of several spins of the spin cluster qubit would also reduce the requirements on the readout sensitivity. For example, the sublattice spin $\left\langle 0\left|\hat{s}_{1, z}+\hat{s}_{3, z}+\hat{s}_{5, z}+\hat{s}_{7, z}+\hat{s}_{9, z}\right| 0\right\rangle \simeq 1$ for $n_{c}=9$ is twice as large as the total magnetic moment $\left\langle 0\left|\hat{S}_{z}\right| 0\right\rangle$ and could be measured after separating the electron spins at even and odd sites of the cluster. This illustrates that readout of single spins is sufficient but not necessary to measure the state of the spin cluster qubit. 
Spin readout can also be effected by optical means. A scheme for gate operation based on Pauli blocking has been suggested recently ${ }^{32}$ In a similar way, the creation of a spin polarized exciton by a circularly polarized laser beam and the subsequent obervation of the reemitted photon would allow one to determine the spin state of a single electron. The underlying principle is that, for a quantum dot with an electron spin pointing up, Pauli's exclusion principle prohibits the creation of an exciton in which the electron with spin up occupies the same orbital state. The creation of an exciton, which can be observed by the photon emitted on recombination, is possible only if the electron spin in the quantum dot points opposite to the one of the exciton created by the laser beam. However, for qubits based on spins in a single quantum dot, the minimum laser focus on the order of the wavelength puts severe constraints on an optical readout scheme. These constraints are relaxed for spin cluster qubits where it is sufficient to have a laser spot size with a diamater smaller than the size of the spin cluster qubit.

\section{B. Varying exchange constants}

The formation of a spin cluster qubit from an odd number of antiferromagnetically coupled spins requires little control over intracluster exchange constants. Although both the energy gap $\Delta$ and matrix elements such as $\left\langle 1\left|\hat{s}_{j, x}\right| 0\right\rangle$ depend on the spatial variation of exchange constants, the general principle of assembling several spins into a cluster qubit remains valid.

In order to demonstrate the robustness of our spin cluster qubit against a variation of exchange constants, we return to the isotropic spin chain but now allow for varying $f_{j}$ in Eq. (3). Because the isotropic spin chain still exhibits a $S=1 / 2$ ground state doublet, ${ }^{21}$ quantum computing is possible as discussed for $f_{j} \equiv 1$ in Sec. 【A From an experimental point of view, a priori knowledge of the factors $f_{j}$ is not necessary for quantum computing. Rather, the relevant matrix elements such as $\left|\left\langle 1\left|\hat{s}_{j, x}\right| 0\right\rangle\right|$ can be determined experimentally. Similarly, a quantum computer could even be assembled from spin cluster qubits which are not identical.

If the exchange constants can be controlled during sample growth, the properties of the spin cluster qubit can be engineered to a certain extent. For clusters with centrosymmetric exchange constants, the time required to perform the square-root of swap gate $U_{*}(\pi / 2)$ for given $J_{*}$ increases with $1 /\left|\left\langle 0\left|\hat{s}_{1, z}\right| 0\right\rangle\right|^{2}$. For $n_{c}=9$ and $f_{j} \equiv 1,\left|\left\langle 0\left|\hat{s}_{1, z}\right| 0\right\rangle\right| \simeq 0.18$ corresponding to an increase in the gate operation time for $U_{*}(\pi / 2)$ by a factor $(0.5 / 0.18)^{2} \simeq 7.7$ compared to the single-spin qubit for given $J_{*}$. However, by tuning the outermost couplings to small values $f_{1}=f_{n_{c}-1} \ll \min _{j=2, \ldots, n_{c}-2} f_{j}$, the spin density at the outermost sites increases and approaches $1 / 3$ [see dashed lines in Fig. 2(d) and Appendix B] for a proof of this statement]. Although the energy gap $\Delta$ also decreases and is approximated by $J f_{1}$ in this limit, a trade-off between the increasing matrix elements and the decreasing energy gap would allow one to decrease gate operation times compared to the chain with spatially constant exchange coupling.

\section{Leakage}

We next discuss in more detail the one qubit rotation gate induced by a transverse magnetic field in order to quantify leakage. A related analysis for the CNOT gate has been reported in Ref. 16 .

Because $\hat{S}_{x}|0\rangle=(\hbar / 2)|1\rangle$ and $\hat{S}_{x}|1\rangle=(\hbar / 2)|0\rangle$, a magnetic field that is uniform over the spin cluster acts on the spin cluster qubit in the same way as on a single spin $s=1 / 2$. In particular, the Hamiltonian

$$
\hat{H}^{\prime}=g \mu_{B} B_{x}(t) \hat{S}_{x}
$$

induces a coherent rotation from $|0\rangle$ to $|1\rangle$ without leakage, as implied by quantum mechanical selection rules. The gate operation time for a rotation by $\phi$ is determined by $\int_{0}^{t} d t^{\prime} g \mu_{B} B_{x}\left(t^{\prime}\right) / \hbar=\phi$ and is identical to the one for single spins. In contrast, the one-qubit rotation effected by a spatially varying magnetic field

$$
\hat{H}^{\prime}=\sum_{j=1}^{n_{c}} g_{j} \mu_{B} B_{j, x}(t) \hat{s}_{j, x}
$$

will in general lead to leakage because of finite matrix elements $\left\langle i\left|\hat{H}^{\prime}\right| 0\right\rangle \neq 0$ and $\left\langle i\left|H^{\prime}\right| 1\right\rangle \neq 0$ coupling the computational basis to higher excited states $|i\rangle \neq|0\rangle,|1\rangle$. The adiabatic theorem guarantees that leakage remains small if the Fourier transform of $B_{j, x}$ vanishes for frequencies larger than $\Delta / \hbar$. Even if this adiabaticity requirement is not met, admixing of higher excited states to $\{|0\rangle,|1\rangle\}$ is controlled by the parameters $g_{j} \mu_{B} B_{j, x} / \Delta$ and remains small if $\left|g_{j} \mu_{B} B_{j, x}\right| \ll \Delta$ for all $j$.

In the following, we concentrate on $n_{c}=5$ spins. As shown in Fig. 3(a) and Fig. (4) a magnetic field constant over the cluster coherently rotates $|0\rangle$ into $|1\rangle$. This is also evidenced by the in-phase rotation of all spins. In order to illustrate that also a spatially inhomogeneous field can induce the one-qubit rotation with high gate fidelity, we now consider a magnetic field $B_{3, x}$ acting only on the central spin $(j=3)$ of the cluster. The field is switched on instantaneously at $t=0$. For $t>0$, the time evolution is then governed by the sum of the time-independent Hamiltonian of the spin cluster, $\hat{H}$, and the Zeeman

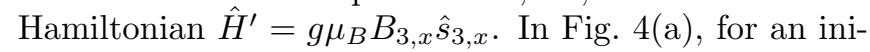
tial state $|\psi(t=0)\rangle=|0\rangle$, we plot the projection of the state $|\psi(t)\rangle$ onto the qubit basis states for $g \mu_{B} B_{3, x} / J=$ 0.1 (dashed line, coinciding with the solid line on this scale) and 0.5 (dashed-dotted line), respectively. The time evolution is obtained by numerical integration of the Schrödinger equation. For small $g \mu_{B} B_{3, x} \ll \Delta \simeq 0.72 J$, the spatially inhomogeneous field rotates $|0\rangle$ into $|1\rangle$ with high fidelity. The gate fidelity $\left|\left\langle 1\left|U_{\text {rot }}\right| 0\right\rangle\right|^{2}$ decreases from 

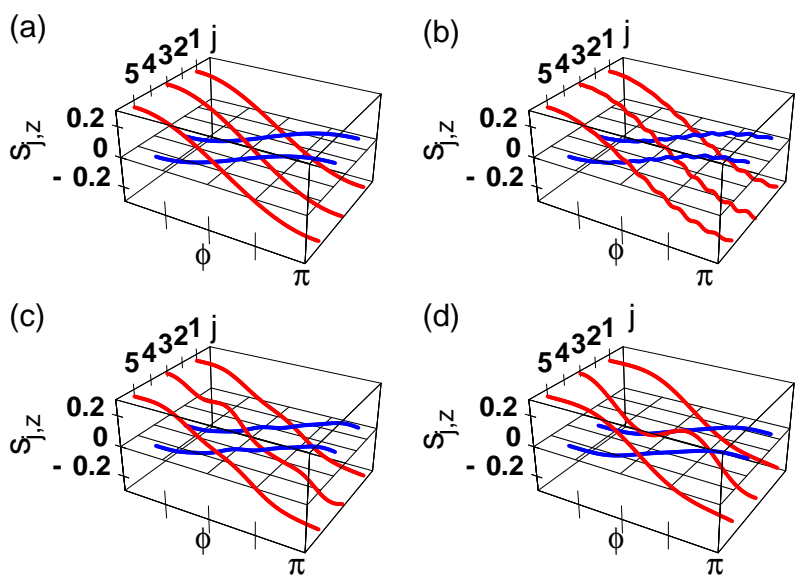

FIG. 3: Local spin density for all sites $j$ of a spin chain with $n_{c}=5$ as a function of time $(\phi \propto t)$ during the onequbit rotation gate. (a) A magnetic field constant over the cluster, $\hat{H}^{\prime}=g \mu_{B} B_{x} \hat{S}_{x}$, coherently rotates $|0\rangle$ into $|1\rangle$ without leakage. Here, $\phi=g \mu_{B} B_{x} t / \hbar$. The coherent rotation is evidenced by the in-phase rotation of all spins. (b)-(d) An inhomogeneous magnetic field, $\hat{H}^{\prime}=g \mu_{B} B_{3, x} \hat{s}_{3, x}$, effects the one-qubit rotation gate with high gate fidelity if $\left|g \mu_{B} B_{3, x}\right| \ll \Delta$. Here, $\phi=g \mu_{B} B_{3, x} t\left(2\left|\left\langle 1\left|\hat{s}_{3, x}\right| 0\right\rangle\right|\right) / \hbar$. The gate fildelity decreases from $99.8 \%$ to $93.4 \%$ and $78.5 \%$ for increasing $g \mu_{B} B_{3, x}=0.1 J(\mathrm{~b}), 0.5 J(\mathrm{c})$, and $1 J(\mathrm{~d})$, respectively. In the local spin density, leakage is evidenced by highfrequency oscillations of neighboring spins, i.e., the excitation of magnons.

$99.8 \%\left(g \mu_{B} B_{x} / J=0.1\right)$ to $93.4 \%$ for $g \mu_{B} B_{x}=0.5 J$, where the typical energy scale of $\hat{H}^{\prime}$ becomes comparable to $\Delta$. Here, $U_{\text {rot }}=\hat{\mathrm{T}}_{t} \exp \left(-i \int_{0}^{t_{\max }} d t\left(\hat{H}+\hat{H}^{\prime}\right) / \hbar\right)$ with $t_{\max }=h /\left(4\left|\left\langle 1\left|\hat{s}_{x, 3}\right| 0\right\rangle\right| g \mu_{B} B_{x}\right)$ describes the time evolution during a $\pi$-rotation.

The decrease in gate fidelity with increasing $B_{3, x}$ can be understood from the local spin densities [Figs. B (b), (c), and (d)]. Although only the central spin is acted on by $B_{3, x}$, for $\left|g \mu_{B} B_{3, x}\right| \ll \Delta$ all spins of the spin cluster corotate with the central spin due to the exchange field. The condition $\left|g \mu_{B} B_{3, x}\right| \ll \Delta$ guarantees that the externally induced rotation of the central spin is sufficiently slow that all spins of the chain corotate in phase. For $\left|g \mu_{B} B_{3, x}\right|$ of order $\Delta$, the rotation of the central spin induced by $B_{3, x}$ is too fast for the remaining spins of the chain to follow in phase [see, e.g., Fig. 31 (c) for short times, $\phi \ll 1]$. The spins of the chain no longer rotate in phase and magnons are excited [Fig. 4(b)]. Quantum gate operation probes the spin dynamics in real time and, hence, may provide new insight into the low energy physics of spin chains. That leakage is controlled by the parameter $\left|g \mu_{B} B_{3, x}\right| / \Delta$ can be traced back to the existence of a ground state doublet.

To illustrate this point, we next contrast our results for a system with antiferromagnetic exchange coupling and a ground state doublet with a ferromagnetic chain with exchange coupling $J<0$ in Eq. (4). For the ferromag-

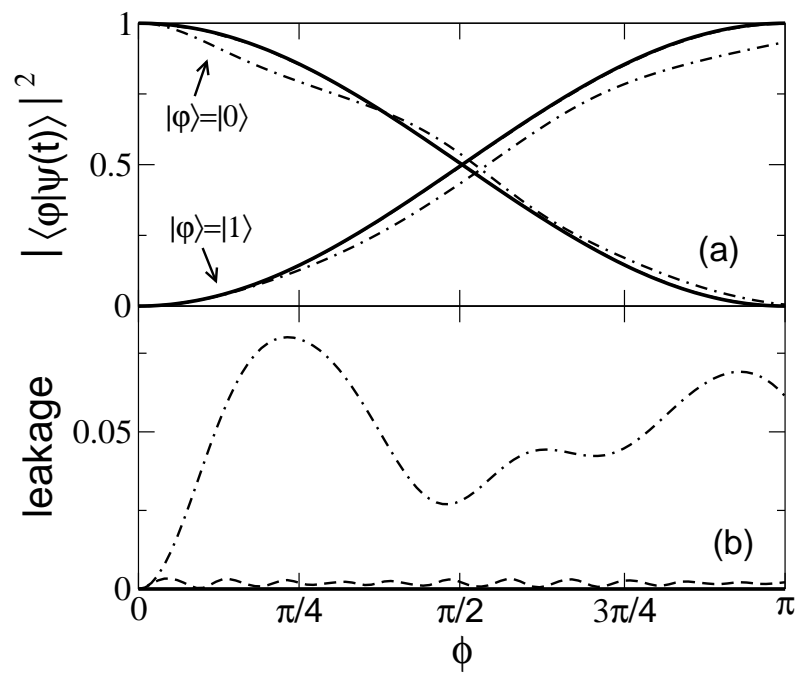

FIG. 4: Time evolution of a state $|\psi(t)\rangle$ with $|\psi(0)\rangle=|0\rangle$ during the one-qubit rotation induced by a spatially constant magnetic field (solid line) and a magnetic field acting only on the central spin of the cluster (dashed and dasheddotted lines) for $n_{c}=5$ as a function of time, $\phi \propto t$. The constant magnetic field effects the quantum gate with $100 \%$ fildelity. In order to illustrate the effect of leakage with increasing Zeeman energy for a spatially inhomogeneous field, $\hat{H}^{\prime}=g \mu_{B} B_{3, x} \hat{s}_{3, x}$, we assume that $\hat{H}^{\prime}$ is switched on instantaneously at $t=0$. (a) For $g \mu_{B} B_{3, x}=0.1 J$ (dashed line), the inhomogeneous magnetic field still effects the one-qubit rotation with $99.8 \%$ fidelity. (b) Leakage out of the computational basis, $1-\left(|\langle 0 \mid \psi(t)\rangle|^{2}+|\langle 1 \mid \psi(t)\rangle|^{2}\right)$, remains smaller than $0.3 \%$. In contrast, for $g \mu_{B} B_{3, x}=0.5 J$ (dashed-dotted lines), i.e., comparable to $\Delta \simeq 0.72 J$, the fidelity is only $93.4 \%$ and leakage is of order $7 \%$.

netic chain, the ground state has degeneracy $n_{c}+1$. A computational basis could be defined also in terms of a subset of the ground state multiplet for $J<0$, e.g., by the states $\left|S_{z}=-n_{c} / 2\right\rangle$ and $\left|S_{z}=-n_{c} / 2+1\right\rangle$. Due to the $n_{c}+1$-fold degeneracy of the ground state multiplet, the system is more prone to leakage than the antiferromagnetic systems considered here because quantum mechanical selection rules no longer prevent transitions from the computational basis to other states in the ground state multiplet.

\section{XY-like chains}

Our considerations have so far been focused on an isotropic exchange coupling which is found, e.g., for coupled quantum dots.$\underline{\underline{5}}$ However, spin cluster qubits could not only be realized in such artificial superstructures. Rather, a wide variety of spin $-1 / 2$ chains in which magnetic ions are coupled by an exchange interaction has been synthesized ${ }^{33}$ The control of single ion spins in a molecule or solid in which the ions are rather closely spaced is even more challenging than the control of a single spin in a quantum dot. Defining a qubit in terms of 
the collective state of a short spin chain will make it substantially simpler to address qubits. However, in contrast to coupled quantum dots, the exchange coupling in many spin chains found in nature is anisotropic ${ }^{33}$ Hence, we next turn to a discussion of anisotropic chains, $J_{\perp} \neq J_{z}$.

For odd $n_{c}$, the spectrum still exhibits a ground state doublet of $\hat{S}_{z}$ eigenstates with eigenvalues $\pm \hbar / 2$, respectively. However, $\hat{\mathbf{S}}^{2}$ is no longer a good quantum number. Both for $J_{\perp} \gg J_{z}$ (XY-like systems) and $J_{\perp} \ll J_{z}$ (Isinglike systems), $|0\rangle$ and $|1\rangle$ can be explicitly constructed.

We first consider the XY model with $J_{z}=0$ in Eq. (3). By the Jordan-Wigner transformation, ${ }^{34}$ the XY chain is mapped onto a system of noninteracting spinless fermions on a lattice with spatially varying hopping amplitudes,

$$
\hat{H}=-\frac{J_{\perp}}{2} \sum_{j=1}^{n_{c}-1} f_{j}\left(\hat{\psi}_{j+1}^{\dagger} \hat{\psi}_{j}+\hat{\psi}_{j}^{\dagger} \hat{\psi}_{j+1}\right),
$$

where

$$
\hat{\psi}_{j}=\exp \left[i \pi \sum_{k=1}^{j-1}\left(\hat{s}_{k, z}+\frac{1}{2}\right)\right] \hat{s}_{j}^{-}
$$

annihilates a Jordan-Wigner fermion at site $j$. The problem is thus reduced to calculating the one-particle eigenenergies and eigenstates of Eq. (28). The oneparticle Hamiltonian has $\left(n_{c}-1\right) / 2$ pairs of states with negative and positive energy $\mp E_{i}$, respectively, which are related to each other by staggering of the wave function,

$$
\mathbf{e}_{i}^{ \pm}=\left(e_{i, 1}, \pm e_{i, 2}, e_{i, 3}, \pm e_{i, 5}, \ldots\right),
$$

where $e_{i, j}$ are real numbers. In addition, there is one eigenstate

$$
\mathbf{e}_{0} \propto\left(1,0,-\frac{f_{1}}{f_{2}}, 0, \frac{f_{1} f_{3}}{f_{2} f_{4}}, 0, \ldots, \pm \frac{f_{1} f_{3} \ldots f_{n_{c}-2}}{f_{2} f_{4} \ldots f_{n_{c}-1}}\right)
$$

with energy eigenvalue 0 . The ground state doublet of the $\mathrm{XY}$ chain corresponds to the lowest $\left(n_{c}-1\right) / 2$ and $\left(n_{c}+\right.$ 1)/2 Jordan-Wigner fermion levels filled. Similarly to the spin chain with isotropic exchange interactions, onequbit gates can be realized by magnetic fields $B_{z}(t)$ and $B_{x}(t)$. By numerical exact diagonalization of small spin chains $\left(n_{c}=9\right)$, we have shown that $\left|\left\langle 1\left|\hat{S}_{x}\right| 0\right\rangle\right|$ remains of order $1 / 2$ for various set of $f_{j}$ [e.g., $f_{j} \equiv 1, f_{j}=$ $\left.\sin \left(j \pi / n_{c}\right)\right]$, such that the operation time for the onequbit rotation gate is only limited by $\hbar / \Delta$. An isotropic interqubit coupling $\hat{H}_{*}=J_{*}(t) \hat{\mathbf{s}}_{n_{c}}^{\mathrm{I}} \cdot \hat{\mathbf{s}}_{1}^{\mathrm{II}}$ for two-qubit gates still translates into the effective Hamiltonian in Eq. (11). With Eqs. (30) and (31), from the completeness relation $\sum_{i=1}^{\left(n_{c}-1\right) / 2}\left(e_{i, j}^{+2}+e_{i, j}^{-2}\right)+e_{0, j}^{2}=1$ for $j=1, \ldots, n_{c}$ and $\hat{s}_{j, z}=\hat{\psi}_{j}^{\dagger} \psi_{j}-1 / 2$, one can calculate all matrix elements entering the effective coupling Hamiltonian Eq. (11),

$$
\begin{aligned}
\left\langle 0\left|\hat{s}_{n_{c}, z}\right| 0\right\rangle & =\frac{e_{0,1}^{2}}{2}, \\
\left|\left\langle 1\left|\hat{s}_{n_{c}, x}\right| 0\right\rangle\right| & =\frac{e_{0,1}}{2},
\end{aligned}
$$

where $e_{0,1}$ is the first component of the normed oneparticle eigenstate defined in Eq. (31). In particular, for $f_{j} \equiv 1,\left\langle 0\left|\hat{s}_{n_{c}, z}\right| 0\right\rangle=1 /\left(n_{c}+1\right)$ and $\left|\left\langle 1\left|\hat{s}_{n_{c}, x}\right| 0\right\rangle\right|=$ $1 / \sqrt{2\left(n_{c}+1\right)}$. Due to the anisotropy of the intrachain exchange coupling, $\hat{H}_{*}$ (which is isotropic in the single-spin operators) translates into an effective XXZHamiltonian in the two-qubit product basis. Nevertheless, the CNOT gate can still be realized according to Eq. (14). For the anisotropic chain, a magnetic field applied along an axis $\mathbf{n}$ translates into a rotation around the axis $\left(n_{x}, n_{y}, n_{z} / 2\left|\left\langle 1\left|\hat{S}_{x}\right| 0\right\rangle\right|\right)$ in the Hilbert space spanned by $\{|0\rangle,|1\rangle\}$,

$$
\begin{aligned}
\hat{H}^{\prime}=g \mu_{B} B \mathbf{n} & \cdot \hat{\mathbf{S}} \\
\simeq g \mu_{B} B 2\left|\left\langle 1\left|\hat{S}_{x}\right| 0\right\rangle\right|\left[n_{x}(|0\rangle\langle 1|+| 1\rangle\langle 0|) / 2\right. & \\
& \left.+n_{y} i(|1\rangle\langle 0|-| 0\rangle\langle 1|) / 2\right] \\
& +g \mu_{B} B n_{z}(|0\rangle\langle 0|-| 1\rangle\langle 1|) / 2 .
\end{aligned}
$$

A one-qubit rotation around an arbitrary axis, e.g. $\mathbf{n}_{1}$ in Eq. (14), hence requires appropriate rescaling of the magnetic fields 16

\section{E. Ising-like chains}

In the Ising limit $J_{z} \gg J_{\perp}$ the ground state doublet

$$
\begin{aligned}
& |0\rangle=|\uparrow\rangle_{1}|\downarrow\rangle_{2} \ldots|\uparrow\rangle_{n_{c}}+\mathcal{O}\left(J_{\perp} / J_{z}\right), \\
& |1\rangle=|\downarrow\rangle_{1}|\uparrow\rangle_{2} \ldots|\downarrow\rangle_{n_{c}}+\mathcal{O}\left(J_{\perp} / J_{z}\right)
\end{aligned}
$$

is separated from the next excited state by an $n_{c^{-}}$ independent $\Delta \sim J_{z} \min \left(f_{j}\right)$. In perturbation theory in $J_{\perp} / J_{z}$, for $f_{j} \equiv 1$, the matrix elements

$$
\begin{aligned}
\left|\left\langle 1\left|\hat{S}_{x}\right| 0\right\rangle\right| & \simeq \frac{n_{c}+1}{4}\left(\frac{2 J_{\perp}}{J_{z}}\right)^{\left(n_{c}-1\right) / 2}, \\
\left|\left\langle 1\left|\hat{s}_{n_{c}, x}\right| 0\right\rangle\right| & \simeq \frac{1}{2}\left(\frac{2 J_{\perp}}{J_{z}}\right)^{\left(n_{c}-1\right) / 2}
\end{aligned}
$$

decrease exponentially with system size because $\hat{S}_{x}$ and $\hat{s}_{n_{c}, x}$ only flip one spin within the chain. Expanding the states $|0\rangle$ and $|1\rangle$ in powers of $J_{\perp} / J_{z}$ it follows that finite matrix element of $\hat{s}_{1, x}$ and $\hat{S}_{x}$ between $|0\rangle$ and $|1\rangle$ occur only in order $\left(n_{c}-1\right) / 2$ in $J_{\perp} / J_{z}, \underline{35}$ Even for medium sized chains $n_{c} \gtrsim 9$ and $J_{\perp} / J_{z}<0.2$, an isotropic interqubit coupling Hamiltonian $\hat{H}_{*}$ translates into an effective Hamiltonian Eq. (11) of Ising form (Fig. 51). Because of the long gate operation times implied by Eq. (36) for the one-qubit rotation and, in particular, the CNOT gate, only quantum computing schemes which require a small number of such operations ${ }^{36}$ appear feasible.

In Fig. 5 for a chain with $n_{c}=9$, we compare our analytical results (solid lines) for the matrix elements $\left|\left\langle 1\left|\hat{S}_{x}\right| 0\right\rangle\right|$ and $\left|\left\langle 1\left|\hat{s}_{1, x}\right| 0\right\rangle\right|$ as functions of anisotropy with exact diagonalization for the chain with $f_{j} \equiv 1$ (symbols). Because matrix elements of order unity imply quantum 


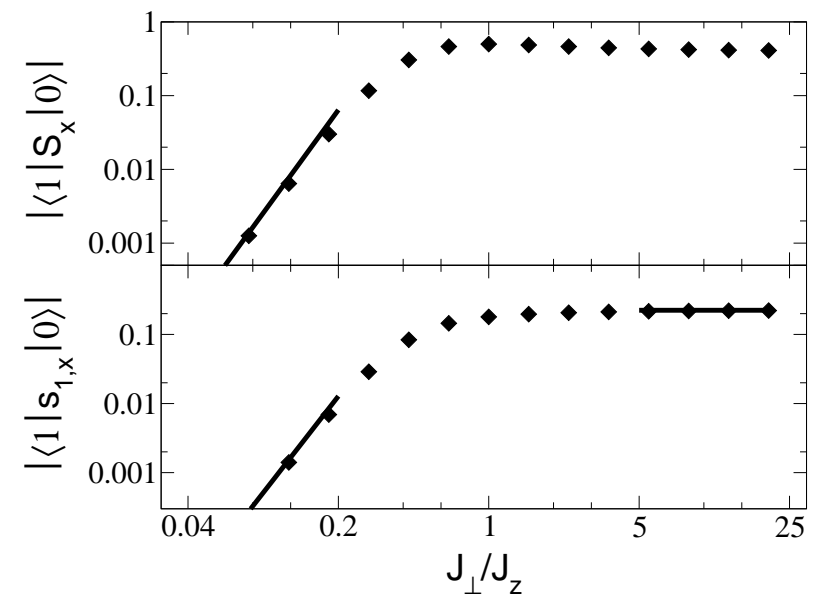

FIG. 5: Transition matrix elements $\left|\left\langle 1\left|\hat{S}_{x}\right| 0\right\rangle\right|$ and $\left|\left\langle 1\left|\hat{s}_{1, x}\right| 0\right\rangle\right|$ as a function of exchange anisotropy $\left(J_{\perp} \neq J_{z}\right)$. The matrix elements determine gate operation times for the one-qubit rotation and CNOT gate. Diamonds show numerical results obtained for $n_{c}=9$ and spatially constant exchange interactions, $f_{j} \equiv 1$, in comparison with analytical results (solid lines) [see Eq. (36)].

gate operation times comparable to single spins, the results in Fig. 5 show that universal quantum computing based on a sequence of one-qubit rotation and CNOT gates is feasible for a wide range of spin cluster qubits.

\section{F. Experimental realization}

We illustrate the advantages of a spin cluster qubit formed by $n_{c}=5$ spins $s=1 / 2$ in a one-dimensional array of quantum dots with diameter $d \simeq 50 \mathrm{~nm}$. For realistic parameters ${ }^{5}\left(J \simeq 10 \mathrm{~K} k_{B}, J_{*} \simeq 2.3 \mathrm{~K} k_{B}\right.$, $\left.g \mu_{B} B \simeq 0.7 \mathrm{~K} k_{B}\right)$ and a magnetic field which decreases from its maximum values $B_{x}$ at the central site of the chain to $0.2 B_{x}$ at sites $j=2$, 4 , the operation time for one-qubit gates increases by a factor $1 / 2 \mid\langle 1| 0.2 \hat{s}_{2, x}+$ $\hat{s}_{3, x}+0.2 \hat{s}_{4, x}|0\rangle \mid \simeq 2.2$ compared to a single spin. The operation time for the square-root of SWAP gate is increased by a factor of $1 /\left(2\left|\left\langle 0\left|\hat{s}_{1, z}\right| 0\right\rangle\right|\right)^{2} \simeq 4$ compared to the single-spin qubit. However, the operation time for the CNOT gate as defined in Eq. (14) is mainly determined by the single-qubit operations of the sequence. Hence, for the minimum operation time of the CNOT gate we find 386 ps for spin clusters instead of 165 ps for single spins. The decrease of decoherence time strongly depends on the microscopic origin of decoherence. From the heuristic argument in Sec. IIA we find that the decoherence rate due to globally fluctuating magnetic fields does not scale with $n_{c}$ and is equal to the one of the single-spin qubit. Decoherence caused by fluctuating local fields scales linearly with $n_{c}$. For a spin cluster qubit with $n_{c}=5$, we estimate that the number of quantum gates which can be performed during the decoherence time decreases by at most a factor of 10 compared to the single-spin qubit. However, in contrast to single-spin qubits, magnetic fields and exchange constants must be controlled only over length scales $n_{c} d=250 \mathrm{~nm}$ and $2 n_{c} d=500 \mathrm{~nm}$, respectively instead of $d=50 \mathrm{~nm}$. This would allow one to control the exchange interaction between neighboring clusters optically ${ }^{6}$ Note that, for the small clusters analyzed here, $\Delta$ is so large that neither adiabaticity nor the requirement that the Zeeman energy and $J_{*}$ be small compared to $\Delta$ provides a serious restriction on quantum gate operation times.

Decoherence due to dipolar interactions is unimportant for electron spins in quantum dots with a typical distance $d=50-100 \mathrm{~nm}$, where $E_{\text {dip }}$ [Eq. (21)] is of order $10^{-11} \mathrm{~K} k_{B}=\hbar / 0.25 s$. However, for $\mathrm{P}$ dopants in a Si matrix with a typical distance $d=100 \AA, E_{\text {dip }}=$ $6.2 \times 10^{-7} \mathrm{~K}_{B}=\hbar / 12 \mu \mathrm{s}$. Recent experiments ${ }^{37}$ show that magnetic dipolar interactions are indeed among the most dominant decoherence mechanisms for $\mathrm{P}$ dopants in a $\mathrm{Si}$ matrix. The characteristic time scale for dephasing by dipolar interactions, $12 \mu \mathrm{s}$, is larger than realistic gate operation times by only a factor $10^{2}-10^{3} .38 .39$ However, for a spin cluster formed by $n_{c}=9$ spins with a dominant nearest neighbor exchange interaction, the intercluster dipolar interaction energy is reduced compared to the one of single spins by a factor of 8 , i.e., the characteristic decoherence time for dipolar interactions increases to $100 \mu \mathrm{s}$.

\section{SPIN CLUSTERS IN $d>2$}

So far, our considerations have been restricted to spin chains. The main ideas discussed above apply to a much larger class of antiferromagnetic systems with uncompensated sublattices. We illustrate next that quantum gates are feasible also if spins $s=1 / 2$ are arranged in a twoor three-dimensional cluster. For definiteness, we restrict our attention to an isotropic exchange interaction $J>0$.

\section{A. Bipartite lattice}

We first consider an odd number of spins arranged on a bipartite lattice with the number of sublattice sites differing by 1 , e.g., a rectangular lattice with $L_{1} \times L_{2}$ sites, where $L_{1,2}$ are odd. This two-dimensional lattice exhibits a ground state doublet ${ }^{21}$. Similarly to the spin chain, the computational basis $\{|0\rangle,|1\rangle\}$ can be defined in terms of the $\hat{S}_{z}$-eigenstates of the ground state doublet. Here, $\hat{\mathbf{S}}$ is the operator of total spin of the two-dimensional array. From a spin-wave ansatz for the elementary excitations, the energy gap $\Delta$ separating the ground state doublet from the next excited state can be estimated as

$$
\Delta \simeq \frac{J \pi}{\min \left(L_{1}, L_{2}\right)}
$$

Because the characteristic features of the ground state doublet carry over from the one- to the two-dimensional 


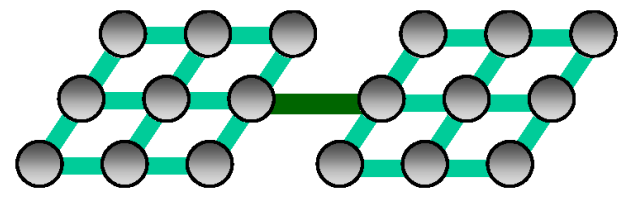

(a)

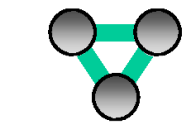

(b)

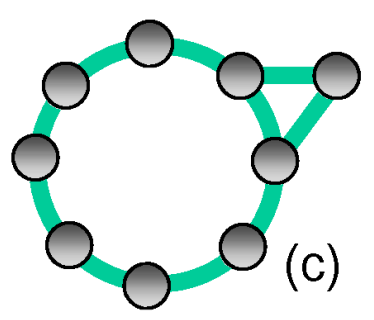

FIG. 6: Two-dimensional spin clusters. Each dot represents a single-spin qubit. (a) The spin cluster qubit scheme is readily extended from spin chains to any bipartite lattice. (b) Spin arrays with frustrated bonds have a highly degenerate ground state (fourfold degeneracy for three spins). (c) If the frustrated bonds are part of a larger array, the high degeneracy is usually lifted and a ground state doublet remains.

spin cluster qubit, quantum computing with twodimensional spin arrays on bipartite lattices is possible with the techniques discussed in Sec. IIA Gate operation times are determined by the matrix elements $\left|\left\langle 0\left|\hat{s}_{j, z}\right| 0\right\rangle\right|=\left|\left\langle 1\left|\hat{s}_{j, x}\right| 0\right\rangle\right|$. For the $3 \times 3$-lattice shown in Fig. [6] (a), from exact diagonalization we find $\left\langle 0\left|\hat{s}_{j, z}\right| 0\right\rangle=$ 0.15 for sites $j$ in the center of the edges, $\left\langle 0\left|\hat{s}_{j, z}\right| 0\right\rangle=0.23$ for sites $j$ at the corners, and $\left\langle 0\left|\hat{s}_{j, z}\right| 0\right\rangle=0.17$ for the central site of the cluster. Similarly to the spin chain, the ground state doublet is robust against a spatial variation in the exchange constants as long as all exchange constants remain antiferromagnetic.

\section{B. Geometrically frustrated systems}

For non-bipartite lattices, a ground-state doublet is not guaranteed to emerge. The simplest example is the geometrically frustrated system of three spins $s=1 / 2$ shown in Fig. 6](b),

$$
\begin{aligned}
\hat{H} & =J\left(\hat{\mathbf{s}}_{1} \cdot \hat{\mathbf{s}}_{2}+\hat{\mathbf{s}}_{1} \cdot \hat{\mathbf{s}}_{3}+\hat{\mathbf{s}}_{2} \cdot \hat{\mathbf{s}}_{3}\right) \\
& =\frac{J}{2}\left(\hat{\mathbf{S}}^{2}-\frac{9}{4}\right)
\end{aligned}
$$

which has a fourfold degenerate ground state with energy eigenvalue $E=-0.75 J$. The eigenstates can be chosen as

$$
\begin{aligned}
& |0\rangle=(|\uparrow \uparrow \downarrow\rangle-|\uparrow \downarrow \uparrow\rangle) / \sqrt{2}, \\
& |1\rangle=(|\downarrow \uparrow \downarrow\rangle-|\downarrow \downarrow \uparrow\rangle) / \sqrt{2}, \\
& |2\rangle=(2|\uparrow \downarrow \downarrow\rangle-|\downarrow \uparrow \downarrow\rangle-|\downarrow \downarrow \uparrow\rangle) / \sqrt{6},
\end{aligned}
$$

$$
|3\rangle=(2|\downarrow \uparrow \uparrow \uparrow-| \uparrow \uparrow \downarrow\rangle-|\uparrow \downarrow \uparrow\rangle) / \sqrt{6}
$$

As demonstrated in Ref. 40, these states could still define a logical qubit robust against certain sources of decoherence. However, quantum gate operation would always require control over single exchange interactions or local magnetic fields and exclude quantum computing with control parameters which vary slowly in space.

Geometrical frustration does, however, not in general rule out the existence of a ground state doublet. In the more generic case that geometrically frustrated bonds are part of a larger system [Fig. 6(c)] or the exchange constants in Fig. 6(b) are not all equal, a ground state doublet emerges. In this case, the logical states of the spin cluster qubit again can be defined in terms of the $\hat{S}_{z}$ eigenstates of the ground state doublet and quantum gate operation is possible with magnetic fields and exchange constants varying slowly over the cluster. For systems as shown in Fig. 6(c), in which some bonds are frustrated, $\Delta$ is usually smaller than in the case of bipartite lattices. For example, for Fig. 6(c), $\Delta=0.157 \mathrm{~J}$ compared to $\Delta=0.991 \mathrm{~J}$ for Fig. 6(a). According to the adiabaticity requirement, the small gap limits gate operation times more severely for the system in Fig. 6] (c).

\section{Experimental realization}

Because spin cluster qubits emerge also in twodimensional regular spin arrays, spin cluster qubits can be arranged in a plane if the positions of single spins can be controlled as, e.g., for lateral quantum dots [Fig.6(a)]. For a spin cluster qubit formed by $L \times L=n_{c}$ quantum dots, $\Delta \propto 1 / L$. Decoherence due to globally fluctuating fields does not increase with $n_{c}$, whereas independent local Gaussian white noise gives rise to a decoherence rate $1 / \tau_{\phi} \propto n_{c}$. Two-dimensional spin cluster qubits are hence particularly interesting for qubits in which decoherence is induced mainly by global rather than local fluctuating fields.

More importantly, a spin cluster qubit can be defined even for a wide range of systems in which the positions and exchange constants cannot be accurately controlled. For $\mathrm{P}$ atom electron spins in a Si matrix, because of rapid oscillations of the exchange coupling between atoms at large distances, placement of atoms with lattice spacing precision is required for single-spin qubits 38 Without this precision, the exchange interaction at large distances vanishes with a probability of $50 \%$. In contrast, for spin cluster qubits formed by a small number (e.g. three) of $\mathrm{P}$ dopants located close to each other, the spin defining the logical state of the qubit is delocalized over the cluster. The effective exchange coupling between neighboring qubits obtained by integration of the exchange interaction over the clusters is finite with a high probability. Because the intracluster exchange interaction at small distances varies strongly with distance, 38 for a random arrangement of three spins, the exchange couplings 
will differ with high probability and the system is not frustrated.

\section{LARGER SPINS}

So far, our considerations have been restricted to clusters formed by spins $s=1 / 2$. We next consider antiferromagnetic systems with larger spins $s>1 / 2$.

\section{A. Antiferromagnetic molecular clusters}

Only very recently it has been shown theoretically that Grover's algorithm can be implemented with ferromagnetic molecular magnets using a unary encoding. ${ }^{41.42}$ In view of universal quantum computing, ferromagnetic clusters such as the molecular magnets $\mathrm{Mn}_{12}$ and $\mathrm{Fe}_{8}$ (Ref. 43) suffer from the large net spin which usually means large matrix elements coupling the spin to the environment and, hence, short decoherence times.

In contrast, in antiferromagnetic systems, such as various ring compounds, 43 the spins couple such that they form a small total magnetic moment. Antiferromagnetic clusters which have unequal sublattice magnetization, will in general have a ground state multiplet rather than the singlet found for systems with compensated sublattice spins ${ }^{44}$ Several antiferromagnetic molecular magnets comprised of spins with quantum numbers larger than $1 / 2$ have been synthesized to date 45.46 .47 .48 .49 including several compounds with uncompensated sublattice spins ${ }^{50}$ As a paradigm, we consider systems with isotropic exchange interaction $J$, but allow for an easy or hard axis single-spin anisotropy,

$$
\hat{H}=J \hat{\mathbf{s}}_{1} \cdot \hat{\mathbf{s}}_{2}+k_{z}\left(\hat{s}_{1, z}^{2}+\hat{s}_{2, z}^{2}\right) .
$$

Here, $s_{1}$ and $s_{2}=s_{1} \pm 1 / 2$ are the spin quantum numbers of the two sublattices, respectively, $J>0$ is an effective exchange constant, and $k_{z}$ the single ion anisotropy. Equation (40) has a ground state doublet $\{|0\rangle,|1\rangle\}$ of $\hat{S}_{z}=\hat{s}_{1, z}+\hat{s}_{2, z}$ eigenstates with eigenvalues $\pm \hbar / 2$, respectively. Because $\left[\hat{S}_{z}, \hat{H}\right]=0$ for the Hamiltonian Eq. (40), the logical qubit basis states have an expansion of the form (for $s_{2}=s_{1}-1 / 2$ )

$$
\begin{aligned}
|0\rangle= & \sum_{m_{1}=-s_{1}+1}^{s_{1}} \alpha_{m_{1}}\left|m_{1}, 1 / 2-m_{1}\right\rangle \\
= & \alpha_{-s_{1}+1}\left|-s_{1}+1, s_{1}-1 / 2\right\rangle \\
& +\alpha_{-s_{1}+2}\left|-s_{1}+2, s_{1}-3 / 2\right\rangle+\ldots \\
& +\alpha_{s_{1}}\left|s_{1},-s_{1}+1 / 2\right\rangle
\end{aligned}
$$

in terms of the spin product basis. For $s_{1,2} \gg 1$, analytical expressions can be derived both for the action of a magnetic field (one-qubit rotation gate) and for the action of an interqubit coupling Hamiltonian (two-qubit gates) between clusters I and II,

$$
\hat{H}_{*}=J_{*}(t) \hat{\mathbf{s}}_{2}^{\mathrm{I}} \cdot \hat{\mathbf{s}}_{1}^{\mathrm{II}}
$$

within a coherent state path integral formalism ${ }^{34,51}$ We only state the main results of our calculations here. Further details are given in Appendix C.

\section{B. Hard axis systems}

For strong hard axis anisotropy, $k_{z}>0$ and $k_{z} s_{1,2}^{2} / J \gg 1$, the spins $\mathbf{s}_{1}$ and $\mathbf{s}_{2}$ lie close to the $x-y$ plane for both states of the ground state doublet. A large contribution in the expansion Eq. (41) comes from the states $\left|m_{1}=0\right\rangle\left|m_{2}=1 / 2\right\rangle$ and $\left|m_{1}=0\right\rangle\left|m_{2}=-1 / 2\right\rangle$, respectively. For illustration, for $s_{1}=3, s_{2}=5 / 2$, and $k_{z} / J=0.2$, by numerical diagonalization of Eq. (40), we find

$$
\begin{aligned}
|0\rangle= & 0.25\left|3,-\frac{5}{2}\right\rangle-0.41\left|2,-\frac{3}{2}\right\rangle \\
& +0.52\left|1,-\frac{1}{2}\right\rangle-0.52\left|0, \frac{1}{2}\right\rangle \\
& +0.42\left|-1, \frac{3}{2}\right\rangle-0.24\left|-2, \frac{5}{2}\right\rangle .
\end{aligned}
$$

The state $|1\rangle$ is obtained by $\left|m_{1}, m_{2}\right\rangle \rightarrow\left|-m_{1},-m_{2}\right\rangle$ on the right hand side of Eq. (43). In agreement with the semiclassical theory, a major contribution to $\{|0\rangle,|1\rangle\}$ comes from states with small $m_{1}$ and $m_{2}$.

In the following, we restrict our attention to systems with large anisotropy, $k_{z}\left(s_{1}^{2}+s_{2}^{2}\right) / J \gg 1$. Then, $\Delta \simeq J$ (Appendix C) and

$$
\begin{aligned}
\left|\left\langle 1\left|\hat{S}_{x}\right| 0\right\rangle\right| & =1 / 4, \\
\left|\left\langle 1\left|\hat{s}_{1, x}\right| 0\right\rangle\right| & =s_{1} / 2, \\
\left|\left\langle 1\left|\hat{s}_{2, x}\right| 0\right\rangle\right| & =s_{2} / 2 .
\end{aligned}
$$

In particular, Eq. (42) translates into the effective Hamiltonian

$$
\begin{gathered}
\hat{H}_{*}=J_{*}\left|\left\langle 0\left|\hat{s}_{1, z}\right| 0\right\rangle\right|\left|\left\langle 0\left|\hat{s}_{2, z}\right| 0\right\rangle\right|\left(\begin{array}{cccc}
1 & 0 & 0 & 0 \\
0 & -1 & 0 & 0 \\
0 & 0 & -1 & 0 \\
0 & 0 & 0 & 1
\end{array}\right) \\
+\frac{J_{*} s_{1} s_{2}}{2}\left(\begin{array}{cccc}
0 & 0 & 0 & 0 \\
0 & 0 & 1 & 0 \\
0 & 1 & 0 & 0 \\
0 & 0 & 0 & 0
\end{array}\right)
\end{gathered}
$$

in the two-qubit product basis. As discussed in Sec. II the CNOT gate can be realized with a unitary time evolution governed by this effective qubit coupling of the XXZ-form.

Matrix elements of order unity in Eq. (44) show that, e.g., a magnetic field $B_{x}$ efficiently rotates the state $|0\rangle$ into $|1\rangle$. This is not a priori evident given the rather complicated representation of the ground state doublet in the single-spin product basis [Eq. (41)]. The large matrix elements arise because, for both $|0\rangle$ and $|1\rangle$, the spins lie close to the $x-y$ plane in the hard axis system. 


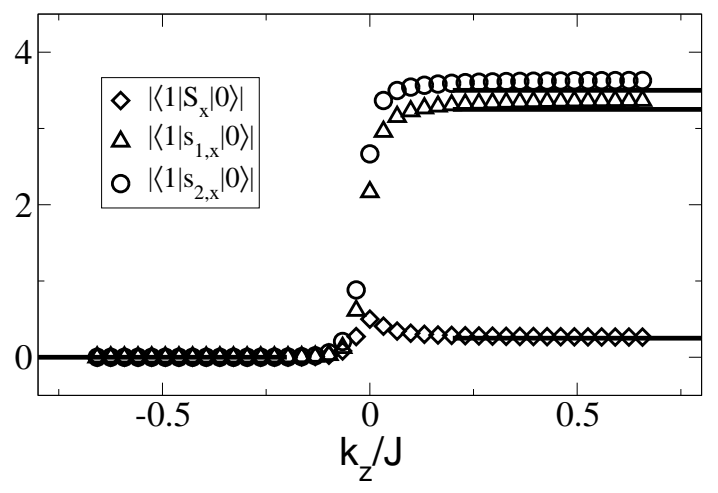

FIG. 7: Matrix elements of spin operators of a qubit formed by two spins with spin quantum numbers $s_{1}=7$ and $s_{2}=6.5$. Numerical data (symbols) obtained from exact diagonalization are in good agreement with analytical results (solid lines).

\section{Easy axis systems}

For $k_{z}<0$, configurations with spins aligned along the z-axis are energetically favorable. We restrict our attention to systems with large anisotropy, $4\left|k_{z}\right|\left(s_{1}^{2}+s_{2}^{2}\right) / J \gg$ 1. Because a transition from $|0\rangle$ to $|1\rangle$ requires a rotation of both spins through a large energy barrier, from the theory of spin quantum tunneling in antiferromagnetic systems $^{52.53}$ we find that $\left|\left\langle 1\left|\hat{S}_{x}\right| 0\right\rangle\right|,\left|\left\langle 1\left|\hat{s}_{1, x}\right| 0\right\rangle\right|,\left|\left\langle 1\left|\hat{s}_{2, x}\right| 0\right\rangle\right| \propto$ $\exp \left(-\sqrt{8\left|k_{z}\right|\left(s_{1}^{2}+s_{2}^{2}\right) / J}\right) \ll 1$ are exponentially small. Similarly to a spin chain in the Ising limit [Sec. IIE], the easy axis system is a candidate for quantum computing schemes as suggested in Ref. 36 .

The analytical results for the matrix elements discussed here are compared with numerical exact diagonalization for $s_{1}=7$ in Fig. 7] We find good agreement with our semiclassical results.

\section{Experimental realization}

Single molecule electrical switches 54.55 .56 .57 have nourished hopes that, in the future, it will be possible to down-scale computers to the level at which bits or qubits are represented by single molecules. The results in Secs. IVB and IVC show that, in such bottom-up approaches aiming at a universal quantum computer, control is not required at the level of single atom spins but only on the scale of molecule spins. In particular, molecular magnetic clusters with an effective spin $S=1 / 2$ define a qubit. One-qubit quantum gates could be effected, for example, by a magnetic tip as used in magnetic force microscopy 58 The spatial resolution of these techniques currently lies in the range of $10-100 \mathrm{~nm}$ (Ref. 59) and approaches the typical size of molecular magnetic clusters 43

Control of the exchange interaction between molecules is challenging. As has been demonstrated recently, ${ }^{55,56}$ the electrical conductivity of individual molecules can be switched between two states in a controlled way. By connecting molecular magnetic clusters by reversible redox switches one could also switch intercluster exchange paths. Alternatively, if the relative position of molecular magnetic clusters can be controlled, the intercluster exchange interaction can be switched on and off via the overlap of electron orbital wave functions by moving clusters relative to each other.

\section{CONCLUSION}

In conclusion, we have shown that quantum computing is possible with a wide variety of clusters assembled from antiferromagnetically coupled spins which form an effective total spin $S=1 / 2$. For arrays of spins $s=1 / 2$, the existence of a spin cluster qubit requires little control over the placement and intracluster coupling of the spins and the spatial dimension of the array. This remains true for a wide range of systems with uncompensated sublattice spins differing by $1 / 2$. We have shown explicitly that, for the spin cluster qubit, initialization, quantum gate operation, and readout are possible with the techniques proposed and analyzed for single spins. The scaling of the decoherence time with system size strongly depends on the microscopic decoherence mechanism. Spin cluster qubits are particularly promising in situations where decoherence is induced mainly by globally fluctuating fields during quantum gate operation and the decoherence rate of the spin cluster qubit is comparable to that of a singlespin qubit or for systems in which magnetic dipolar interactions are the dominant decoherence mechanism. The main advantage of spin clusters compared to single spins is that requirements on local control of magnetic fields and exchange interactions can be traded for longer gate operation times. We have illustrated the feasibility and advantages of spin cluster qubits for arrays of quantum dots, $\mathrm{P}$ dopants in a Si matrix, and molecular magnetic clusters.

In contrast to single spins, spin clusters are not intrinsically two-state systems and leakage during quantum gate operation must be accounted for. For the one-qubit rotation gate, we have shown that leakage is small if the magnetic field which induces the rotation is switched on and off adiabatically or if the Zeeman energy remains small compared to $\Delta$.

Finally, we note that, because any qubit can be mapped onto a spin $s=1 / 2$, the results of this work do not only apply to quantum computing proposals based on spin degrees of freedom but to any quantum computing scheme. More specifically, for any qubit for which methods for initialization, quantum gate operation, quantum error correction, and readout have been identified, a cluster qubit can be formed by coupling several qubits. For the cluster qubit, initialization, quantum gate operation, quantum error correction, and readout are possible using the same techniques as for the original qubit. 


\section{Acknowledgments}

This work was financially supported by the EU TMR network MOLNANOMAG, no. HPRN-CT-1999-00012 (FM and DL), DARPA SPINS and QuIST (JL and DL), the Swiss NCCR Nanoscience (DL), and the Swiss NSF (DL). We acknowledge discussions with G. Burkard, V. Cerletti, W. Coish, H. Gassmann, F. Marquardt, and P. Recher.

\section{APPENDIX A: EFFECTIVE EXCHANGE HAMILTONIAN}

Here, we derive explicitly the effective coupling Hamiltonian in Eq. (11) from Eq. (10). The first and second term in Eq. (11) result from $J_{*}(t) \hat{\mathbf{s}}_{n_{c}}^{\mathrm{I}} \cdot \hat{\mathbf{s}}_{1}^{\mathrm{II}}$ in the microscopic coupling. Decomposing $\hat{\mathbf{s}}_{n_{c}}^{\mathrm{I}} \cdot \hat{\mathbf{s}}_{1}^{\mathrm{II}}=\hat{s}_{n_{c}, z}^{\mathrm{I}} \hat{s}_{1, z}^{\mathrm{II}}+$ $\left(\hat{s}_{n_{c}}^{\mathrm{I}+} \hat{s}_{1}^{\mathrm{II}-}+\hat{s}_{n_{c}}^{\mathrm{I}-} \hat{s}_{1}^{\mathrm{II}+}\right) / 2$ in terms of spin ladder operators, one can readily evaluate the matrix elements in the two-qubit product basis. Because, by definition, $\hat{S}_{z}|0\rangle=(\hbar / 2)|0\rangle$ and $\hat{S}_{z}|1\rangle=(-\hbar / 2)|1\rangle$ and $\hat{s}_{n_{c}, z}^{\mathrm{I}} \hat{s}_{1, z}^{\mathrm{II}}$ conserves the $z$-component of total spin in each cluster separately it follows that

$$
{ }_{\mathrm{I}}\left\langle\left. 0\right|_{\mathrm{II}}\left\langle 1\left|\hat{s}_{n_{c}, z}^{\mathrm{I}} \hat{s}_{1, z}^{\mathrm{II}}\right| 1\right\rangle_{\mathrm{I}} \mid 0\right\rangle_{\mathrm{II}}=0 .
$$

Similarly, all other off-diagonal elements of $\hat{s}_{n_{c}, z}^{\mathrm{I}} \hat{s}_{1, z}^{\mathrm{II}}$ vanish. Because of

$$
{ }_{\mathrm{I}}\left\langle 0\left|\hat{s}_{n_{c}}^{\mathrm{I}}\right| 0\right\rangle_{\mathrm{I}}={ }_{\mathrm{I}}\left\langle 1\left|\hat{s}_{n_{c}}^{\mathrm{I}}\right| 1\right\rangle_{\mathrm{I}}=0,
$$

the transverse exchange interaction $J_{*}(t)\left(\hat{s}_{n_{c}}^{\mathrm{I}+} \hat{s}_{1}^{\mathrm{II}-}+\right.$ $\left.\hat{s}_{n_{c}}^{\mathrm{I}-} \hat{s}_{1}^{\mathrm{II}+}\right) / 2$ has finite matrix elements only between the states $|0\rangle_{\mathrm{I}}|1\rangle_{\mathrm{II}}$ and $|1\rangle_{\mathrm{I}}|0\rangle_{\mathrm{II}}$. This completes the proof that the intercluster exchange term $J_{*}(t) \hat{\mathbf{s}}_{n_{c}}^{\mathrm{I}} \cdot \hat{\mathbf{s}}_{1}^{\mathrm{II}}$ leads to the first and second term in Eq. (11).

It remains to show that a possible change in intracluster exchange interaction constants during two-qubit gate operation, $J_{*}(t) \sum_{j=1}^{n_{c}-1}\left(v_{j}^{\mathrm{I}} \hat{\mathbf{s}}_{j}^{\mathrm{I}} \cdot \hat{\mathbf{s}}_{j+1}^{\mathrm{I}}+v_{j}^{\mathrm{II}} \hat{\mathbf{s}}_{j}^{\mathrm{II}} \cdot \hat{\mathbf{s}}_{j+1}^{\mathrm{II}}\right)$, only leads to a term proportional to $\mathbf{1}$ in Eq. (11). This term conserves all components of the total spin of clusters I and II,

$$
\left[\hat{S}_{\alpha}^{\mathrm{I}}, \sum_{j=1}^{n_{c}-1} v_{j}^{\mathrm{I}} \hat{\mathbf{s}}_{j}^{\mathrm{I}} \cdot \hat{\mathbf{s}}_{j+1}^{\mathrm{I}}\right]=0
$$

for $\alpha=x, y, z$, and similarly for II. Hence, all off-diagonal matrix elements such as ${ }_{\mathrm{I}}\left\langle 1\left|v_{j}^{\mathrm{I}} \hat{\mathbf{s}}_{j}^{\mathrm{I}} \cdot \hat{\mathbf{s}}_{j+1}^{\mathrm{I}}\right| 0\right\rangle_{\mathrm{I}}$ vanish. Finally, because $|1\rangle_{\mathrm{I}}=\hat{S}^{\mathrm{I}-}|0\rangle_{\mathrm{I}}=2 \hat{S}_{x}^{\mathrm{I}}|0\rangle_{\mathrm{I}}$, with Eq. (A3),

$$
\begin{aligned}
& { }_{\mathrm{I}}\left\langle 1\left|{ }_{\mathrm{II}}\left\langle 0\left|J_{*}(t) \sum_{j=1}^{n_{c}-1} v_{j}^{\mathrm{I}} \hat{\mathbf{s}}_{j}^{\mathrm{I}} \cdot \hat{\mathbf{s}}_{j+1}^{\mathrm{I}}\right| 1\right\rangle_{\mathrm{I}}\right| 0\right\rangle_{\mathrm{II}} \\
& \quad={ }_{\mathrm{I}}\left\langle 0\left|{ }_{\mathrm{II}}\left\langle 0\left|2 \hat{S}_{x}^{\mathrm{I}} J_{*}(t) \sum_{j=1}^{n_{c}-1} v_{j}^{\mathrm{I}} \hat{\mathbf{s}}_{j}^{\mathrm{I}} \cdot \hat{\mathbf{s}}_{j+1}^{\mathrm{I}} 2 \hat{S}_{x}^{\mathrm{I}}\right| 0\right\rangle_{\mathrm{I}}\right| 0 \hat{} \mathrm{A} 44\right)
\end{aligned}
$$

$$
\begin{aligned}
& ={ }_{\mathrm{I}}\left\langle\left. 0\right|_{\mathrm{II}}\left\langle 0\left|J_{*}(t) \sum_{j=1}^{n_{c}-1} v_{j}^{\mathrm{I}} \hat{\mathbf{s}}_{j}^{\mathrm{I}} \cdot \hat{\mathbf{s}}_{j+1}^{\mathrm{I}}\left(2 \hat{S}_{x}^{\mathrm{I}}\right)^{2}\right| 0\right\rangle_{\mathrm{I}} \mid 0\right\rangle_{\mathrm{II}} \\
& ={ }_{\mathrm{I}}\left\langle\left. 0\right|_{\mathrm{II}}\left\langle 0\left|J_{*}(t) \sum_{j=1}^{n_{c}-1} v_{j}^{\mathrm{I}} \hat{\mathbf{s}}_{j}^{\mathrm{I}} \cdot \hat{\mathbf{s}}_{j+1}^{\mathrm{I}}\right| 0\right\rangle_{\mathrm{I}} \mid 0\right\rangle_{\mathrm{II}} \cdot
\end{aligned}
$$

In the second line of Eq. A4 we have invoked that $\left\{|0\rangle_{\mathrm{I}},|1\rangle_{\mathrm{I}}\right\}$ belong to one spin-1/2 doublet, the third line then follows from Eq. A3). With a similar argument it can be shown that all diagonal matrix elements in the two-qubit product basis are equal and $J_{*}(t) \sum_{j=1}^{n_{c}-1}\left(v_{j}^{\mathrm{I}} \hat{\mathbf{s}}_{j}^{\mathrm{I}}\right.$. $\left.\hat{\mathbf{s}}_{j+1}^{\mathrm{I}}+v_{j}^{\mathrm{II}} \hat{\mathbf{s}}_{j}^{\mathrm{II}} \cdot \hat{\mathbf{s}}_{j+1}^{\mathrm{II}}\right)$ translates into a term $J_{o}(t) \mathbf{1}$ in the effective coupling Hamiltonian Eq. (11).

Finally, we prove Eq. (13) which implies that, for isotropic intracluster exchange interactions, the effective two-qubit Hamiltonian is also of Heisenberg form. For simplicity, we omit the label I of the spin cluster qubit in the following. In order to formally calculate $\left\langle 0\left|\hat{s}_{n_{c}, z}\right| 0\right\rangle$ and $\left|\left\langle 1\left|\hat{s}_{n_{c}, x}\right| 0\right\rangle\right|$, we define the spin operators

$$
\hat{S}_{\alpha}^{\prime}=\hat{S}-\hat{s}_{n_{c}, \alpha}=\sum_{j=1}^{n_{c}-1} \hat{s}_{j, \alpha}
$$

of all but the outermost spin $j=n_{c}$ of the cluster. Generally, $|0\rangle$ can be expanded as

$$
|0\rangle=a|\Psi\rangle|\uparrow\rangle+b|\Phi\rangle|\downarrow\rangle,
$$

where $|\Psi\rangle$ and $|\Phi\rangle$ describe the normed states of the leftmost $n_{c}-1$ spins in the array and $a$ and $b$ are real numbers. Because $\hat{S}_{z}|0\rangle=(\hbar / 2)|0\rangle,|\Psi\rangle$ and $|\Phi\rangle$ are eigenstates of $\hat{S}_{z}^{\prime}$ with eigenvalues 0 and $\hbar$, respectively. $\hat{S}_{x}|0\rangle=(\hbar / 2)|1\rangle$ is an $\hat{S}_{z}$ eigenstate with eigenvalue $-(\hbar / 2)$, such that $b|\Phi\rangle=-a \hat{S}_{+}^{\prime}|\Psi\rangle$, and

$$
\begin{aligned}
|0\rangle & =a\left(|\Psi\rangle|\uparrow\rangle-\hat{S}_{+}^{\prime}|\Psi\rangle|\downarrow\rangle\right), \\
|1\rangle & =a\left(\hat{S}_{-}^{\prime}|\Psi\rangle|\uparrow\rangle-\left(1-\hat{S}_{-}^{\prime} \hat{S}_{+}^{\prime}\right)|\Psi\rangle|\downarrow\rangle\right), \\
a & =\frac{1}{\sqrt{1+\left\langle\Psi\left|\hat{S}_{-}^{\prime} \hat{S}_{+}^{\prime}\right| \Psi\right\rangle}} .
\end{aligned}
$$

From Eq. A7 we calculate

$$
\begin{aligned}
& \left\langle 0\left|\hat{s}_{n_{c}, z}\right| 0\right\rangle=-\left\langle 1\left|\hat{s}_{n_{c}, z}\right| 1\right\rangle=\frac{1}{2} \frac{1-\left\langle\Psi\left|\hat{S}_{-}^{\prime} \hat{S}_{+}^{\prime}\right| \Psi\right\rangle}{1+\left\langle\Psi\left|\hat{S}_{-}^{\prime} \hat{S}_{+}^{\prime}\right| \Psi\right\rangle}, \\
& \left\langle 1\left|\hat{s}_{n_{c}, x}\right| 0\right\rangle=\frac{1}{2} \frac{1-\left\langle\Psi\left|\hat{S}_{-}^{\prime} \hat{S}_{+}^{\prime}\right| \Psi\right\rangle}{1+\left\langle\Psi\left|\hat{S}_{-}^{\prime} \hat{S}_{+}^{\prime}\right| \Psi\right\rangle}
\end{aligned}
$$

which proves Eq. (13).

\section{APPENDIX B: ISOTROPIC CHAIN WITH SPATIALLY VARYING EXCHANGE INTERACTION}

The local spin density in the energy eigenstates of Eq. (3) depends sensitively on spatial variations of the 
exchange interaction [Fig. 2(d)]. Whereas for $f_{j} \equiv 1$ (solid lines) the magnetization density in each of the sublattices increases toward the center of the chain, 60 the opposite behavior is observed for an exchange interaction $f_{j}=\sin \left(j \pi / n_{c}\right)$ (dashed lines). In the limit $f_{1}=f_{n_{c}-1} \ll \min _{j=2, \ldots, n_{c}-2} f_{j}$, the increase of local spin density toward the ends of the chain can be understood quantitatively. The ground state doublet of the spin cluster qubit can be constructed explicitly from the ground state doublet $\left\{|0\rangle_{n_{c}-2},|1\rangle_{n_{c}-2}\right\}$ of the chain with the outermost spins removed. For $J f_{1}$ much smaller than the energy gap $\Delta_{n_{c}-2}$ of the chain formed by the $n_{c}-2$ central spins, the coupling of the outermost spins can be treated perturbatively. For the chain with centrosymmetric exchange couplings, $f_{j}=f_{n_{c}-j}$, from the ansatz

$$
\begin{aligned}
|0\rangle= & \alpha_{1}|\uparrow\rangle|1\rangle_{n_{c}-2}|\uparrow\rangle+\alpha_{2}|\uparrow\rangle|0\rangle_{n_{c}-2}|\downarrow\rangle \\
& +\alpha_{3}|\downarrow\rangle|0\rangle_{n_{c}-2}|\uparrow\rangle+\mathcal{O}\left(J f_{1} / \Delta_{n_{c}-2}\right),
\end{aligned}
$$

we find $\left(\alpha_{1}, \alpha_{2}, \alpha_{3}\right)=(2,-1,-1) / \sqrt{6}$ for the ground state of the chain with $n_{c}$ spins and, hence,

$$
\lim _{f_{1}=f_{n_{c}-1} \rightarrow 0}\left|\left\langle 0\left|\hat{s}_{1, z}\right| 0\right\rangle\right|=1 / 3
$$

\section{APPENDIX C: LARGE SPINS}

The matrix elements in Eq. (44) can be calculated from coherent state spin path integrals .51 We focus on strong easy plane systems, $k_{z}>0$ and $k_{z}\left(s_{1}^{2}+s_{2}^{2}\right) / J \gg 1$. Following the standard approach for antiferromagnetic systems, the partition function of the two-spin system is expressed as path integral over the Néel vector $\mathbf{n}$ and homogeneous magnetization $\mathbf{l}$ defined by $\mathbf{s}_{1}=s_{1} \mathbf{n}+\mathbf{l}$ and $\mathbf{s}_{2}=-s_{2} \mathbf{n}+\mathbf{l}$, where $\mathbf{n} \cdot \mathbf{l}=0$. Integrating out $\mathbf{l}$ in a saddle point approximation and parametrizing

$$
\mathbf{n}=\left(\begin{array}{c}
\sin \theta \cos \phi \\
\sin \theta \sin \phi \\
\cos \theta
\end{array}\right)
$$

the Euclidean action of the system can be written as 52,53

$$
L_{E}=\frac{\hbar^{2}}{2 J}\left(\dot{\theta}^{2}+\sin ^{2} \theta \dot{\phi}^{2}\right)+k_{z}\left(s_{1}^{2}+s_{2}^{2}\right) \cos ^{2} \theta
$$

$$
+i \Delta s \hbar \dot{\phi}(1-\cos \theta)
$$

where the last factor accounts for the difference $\Delta s=$ $s_{2}-s_{1}$ of the spin quantum numbers and $\dot{\phi}=\partial_{\tau} \phi$ is the imaginary time derivative. In the limit of strong anisotropy, $k_{z}\left(s_{1}^{2}+s_{2}^{2}\right) / J \gg 1$, Eq. (C2) can be expanded to second order in $\theta-\pi / 2$ and the fluctuations are integrated out, leading to

$$
Z=\int \mathcal{D} \phi \exp \left(-\int_{0}^{\hbar \beta} d \tau L_{E}[\phi] / \hbar\right)
$$

with an effective Euclidean Lagrangean

$$
L_{E}[\phi]=\frac{\hbar^{2} \dot{\phi}^{2}}{2 J}+i \Delta s \hbar \dot{\phi} .
$$

After continuation to real time, by a canonical transformation we obtain the Hamiltonian of the system in terms of the Néel vector operator,

$$
\hat{H}=\frac{J}{2 \hbar^{2}}\left(\hat{p}_{\phi}-\hbar \Delta s\right)^{2},
$$

where

$$
\hat{\mathbf{n}}=\left(\begin{array}{c}
\cos \hat{\phi} \\
\sin \hat{\phi} \\
0
\end{array}\right), \hat{\mathbf{l}}=\frac{1}{2}\left(\begin{array}{c}
0 \\
0 \\
\hat{p}_{\phi} / \hbar-\Delta s
\end{array}\right)
$$

and $\hat{p}_{\phi}$ is the momentum operator conjugate to the inplane polar angle $\phi,\left[\hat{p}_{\phi}, \hat{\phi}\right]=-i \hbar$. By inspection of Eq. (C5), we find that the spin system Eq. (40) has been mapped onto the Hamiltonian of a particle on a ring threaded by a magnetic flux $\propto \Delta s$. In particular, for half-integer $\Delta s$, the Hamiltonian has a ground state doublet $\{|0\rangle,|1\rangle\}$ with wave functions $\psi_{0}(\phi)=$ $\exp (i(m+1) \phi) / \sqrt{2 \pi}$ and $\psi_{1}(\phi)=\exp (i m \phi) / \sqrt{2 \pi}$, where $m=\lfloor\Delta s\rfloor$. From $\hat{s}_{1, x} \simeq s_{1} \cos \hat{\phi}$ and $\hat{s}_{2, x} \simeq-s_{2} \cos \hat{\phi}$, one immediately obtains Eq. (44).
1 For a review see e.g. C. H. Bennett and D. P. DiVincenzo, Nature 404, 247 (2000) and references therein.

2 D. P. DiVincenzo, Science 270, 255 (1995).

3 J. I. Cirac and P. Zoller, Nature 406, 579 (2000).

4 D. Loss and D. P. DiVincenzo, Phys. Rev. A 57, 120 (1998).

5 G. Burkard, D. Loss, and D. P. DiVincenzo, Phys. Rev. B 59, 2070 (1999).

6 J. Levy, Phys. Rev. A 64, 052306 (2001).

7 V. Privman, I. D. Vagner, and G. Kventsel, Phys. Lett. A
239, 141 (1998).

8 B. E. Kane, Nature 393, 133 (1998).

9 J. M. Kikkawa, I. P. Smorchkova, N. Samarath, and D. D. Awschalom, Science 277, 1284 (1997).

10 G. Feher, D. K. Wilson, and E. A. Gere, Phys. Rev. Lett. 3, 25 (1959).

11 D. P. DiVincenzo, D. Bacon, J. Kempe, G. Burkard, and K. B. Whaley, Nature 408, 339 (2000).

12 J. Levy, Phys. Rev. Lett. 89, 147902 (2002).

13 D. A. Lidar and L.-A. Wu, Phys. Rev. Lett. 88, 017905 
(2002).

14 D. Bacon, K. R. Brown, and K. B. Whaley, Phys. Rev. Lett. 87, 247902 (2001).

15 S. C. Benjamin, Phys. Rev. Lett. 88, 017904 (2002).

${ }^{16}$ F. Meier, J. Levy, and D. Loss, Phys. Rev. Lett. 90, 047901 (2003).

17 A. Barenco, C. H. Bennett, R. Cleve, D. P. DiVincenzo, N. Margolus, P. Shor, T. Sleator, J. A. Smolin, and H. Weinfurter, Phys. Rev. A 52, 3457 (1995).

18 R. Orbach, Phys. Rev. 112, 309 (1958).

19 L. R. Walker, Phys. Rev. 116, 1089 (1959).

20 E. Lieb, T. Schultz, and D. Mattis, Ann. Phys. (N.Y.) 16, 407 (1961).

21 E. Lieb and D. Mattis, Journ. Math. Phys. 3, 749 (1962).

22 J. C. Bonner and M. E. Fisher, Phys. Rev. 135, A640 (1961).

23 N. Nagaosa, Quantum Field Theory in Strongly Correlated Electronic Systems (Springer, New York, 1998).

24 A. V. Khaetskii, D. Loss, and L. Glazman, Phys. Rev. Lett. 88, 186802 (2002).

25 I. A. Merkulov, Al. L. Efros, and M. Rosen, Phys. Rev. B 65, 205309 (2002).

26 J. Schliemann, A. V. Khaetskii, and D. Loss, Phys. Rev. B 66, 245303 (2002).

27 A. Khaetskii, D. Loss, and L. Glazman, Phys. Rev. B 67, 195329 (2003).

${ }^{28}$ R. de Sousa and S. Das Sarma, Phys. Rev. B 67, 033301 (2003).

29 K. Blum, Density Matrix Theory and Applications (Plenum Press, New York, 1996).

30 W. G. Unruh, Phys. Rev. A 51, 992 (1995).

31 P. Recher, E. V. Sukhorukov, and D. Loss, Phys. Rev. Lett. 85, 1962 (2000).

32 E. Pazy, E. Biolatti, T. Calarco, I. D'Amico, P. Zanardi, F. Rossi, and P. Zoller, Eur. Phys. Lett. 62, 175 (2003).

33 H.-J. Mikeska and M. Steiner, Adv. Phys. 40, 191 (1991).

34 A. Auerbach, Interacting Electrons and Quantum Magnetism (Springer, New York, 1994).

35 J. Villain, Physica B 79, 1 (1975).

${ }^{36}$ R. Raussendorf and H. J. Briegel, Phys. Rev. Lett. 86, 5188 (2001).

37 A. M. Tyryshkin, S. A. Lyon, A. V. Astashkin, and A.M. Raitsimring, cond-mat/0303006

38 B. Koiller, X. Hu, and S. Das Sarma, Phys. Rev. Lett. 88, 027903 (2002).

39 M. Friesen, P. Rugheimer, D. E. Savage, M. G. Lagally, D. W. van der Weide, R. Joynt, and M. A. Eriksson, Phys. Rev. B 67, 121301 (2003).

40 J. Kempe, D. Bacon, D. A. Lidar, and K. B. Whaley, Phys.
Rev. A 63, 042307 (2001).

41 M. N. Leuenberger and D. Loss, Nature 410, 789 (2001).

42 M. N. Leuenberger, D. Loss, M. Poggio, and D. D. Awschalom, Phys. Rev. Lett. 89, 207601 (2002).

43 D. Gatteschi, A. Caneschi, L. Pardi, and R. Sessoli, Science 265, 1054 (1994).

44 F. Meier and D. Loss, Phys. Rev. B 64, 224411 (2001).

${ }^{45}$ K. L. Taft, C. D. Delfs, G. C. Papaefthymiou, S. Foner, D. Gatteschi, and S. J. Lippard, J. Am. Chem. Soc. 116, 823 (1994).

46 A. Caneschi, A. Cornia, A. C. Fabretti, S. Foner, D. Gatteschi, R. Grandi, and L. Schenetti, Chem. Eur. J. 2, 1379 (1996).

47 O. Waldmann, J. Schülein, R. Koch, P. Müller, I. Bernt, R. W. Saalfrank, H. P. Andres, H. U. Güdel, and P. Allenspach, Inorg. Chem. 38, 5879 (1999).

48 O. Waldmann, R. Koch, S. Schromm, J. Schülein, P. Müller, I. Bernt, R. W. Saalfrank, and F. Hampel, Inorg. Chem. 40, 2986 (2001).

49 J. van Slageren, R. Sessoli, D. Gatteschi, A. A. Smith, M. Helliwell, R. E. P. Winpenny, A. Cornia, A. L. Barra, A. G. M. Jansen, E. Rentschler, and G. A. Timco, Chem. Eur. J. 8, 277 (2002).

50 F. K. Larsen, E. J. L. McInnes, H. El Mkami, J. Overgaard, S. Piligkos, G. Rajaraman, E. Rentschler, A. A. Smith, G. M. Smith, V. Boote, M. Jennings, G. A. Timco, and R. E. P. Winpenny, Angew. Chem. Int. Edit. 42, 101 (2003).

51 J. R. Klauder, Phys. Rev. D 19, 2349 (1979).

52 B. Barbara and E. M. Chudnovsky, Phys. Lett. A 145, 205 (1990).

53 I. V. Krive and O. B. Zaslawski, J. Phys. Cond. Matt. 2, 9457 (1990).

54 P. Ball, Nature 406, 118 (2000).

55 C. P. Collier, E. W. Wong, M. Belohradský, F. M. Raymo, J. F. Stoddart, P. J. Kuekes, R. S. Williams, and J. R. Heath, Science 285, 391 (1999).

56 D. I. Gittins, D. Bethell, D. J. Schiffrin, and R. J. Nichols, Nature 408, 67 (2000).

57 C. Joachim, J. K. Gimzewski, and A. Aviram, Nature 408, 541 (2000).

58 D. Rugar, J. Appl. Phys. 68, 1169 (1990).

59 Z. Liu, Y. Danm, Q. Jinjun, and Y. Wu, J. Appl. Phys. 91, 8843 (2002).

60 On a mean field level, the sinusoidal variation of the magnetization density on each of the sublattices can be understood qualitatively from the theory for long chains, S. Eggert and I. Affleck, Phys. Rev. B 46, 10866 (1992). 